\title{
Online Matching with Stochastic Rewards: Optimal Competitive Ratio via Path Based Formulation
}

\author{
Vineet Goyal \\ Columbia University, vgoyal@ieor.columbia.edu \\ Rajan Udwani \\ UC Berkeley, rudwani@berkeley.edu
}

\begin{abstract}
The problem of online matching with stochastic rewards is a generalization of the online bipartite matching problem where each edge has a probability of success. When a match is made it succeeds with the probability of the corresponding edge. Introducing this model, Mehta and Panigrahi (2012) focused on the special case of identical edge probabilities. Comparing against a deterministic offline LP, they showed that the Ranking algorithm of Karp et al. (STOC 1990) is 0.534 competitive and proposed a new online algorithm with an improved guarantee of 0.567 for vanishingly small probabilities. For the case of vanishingly small but heterogeneous probabilities, Mehta et al. (2015) gave a 0.534 competitive algorithm against the same LP benchmark.
\end{abstract}

We consider the more general vertex-weighted version of the problem and give two new results. First, we show that a natural generalization of the Perturbed-Greedy algorithm of Aggarwal et al. (2011), is (1-1/e) competitive when probabilities decompose as a product of two factors, one corresponding to each vertex of the edge. This is the best achievable guarantee as it includes the case of identical probabilities and in particular, the classical online bipartite matching problem. Second, we give a deterministic 0.596 competitive algorithm for the previously well studied case of fully heterogeneous but vanishingly small edge probabilities. A key contribution of our approach is the use of novel path-based formulations and a generalization of the primal-dual scheme of Devanur et al. (2013). These allow us to compare against the natural benchmarks of adaptive offline algorithms that know the sequence of arrivals and the edge probabilities in advance, but not the outcomes of potential matches. These ideas may be of independent interest in other online settings with post-allocation stochasticity.

Key words: Online Matching, Stochastic Rewards, Competitive Ratio, Path Based Analysis, Primal-Dual

\section{Introduction}

Online bipartite matching and its variants and generalizations have been intensely studied for past few decades (Mehta et al. 2013). Internet advertising is a major application domain for these problems, along with Crowdsourcing (Ho and Vaughan 2012, Karger et al. 2014), personalized recommendations (Golrezaei et al. 2014, Ma and Simchi-Levi 2018), ridesharing (Ashlagi et al. 2019, Aouad and Saritaç 2020, Feng et al. 2021), online order order fulfillment (Acimovic and Farias 2019) and many others. Despite a rich body of work on these problems, there are several basic questions that remain open. In this work, we study one such problem. To introduce our setting, we start with the classic online bipartite matching problem. 
Online bipartite matching (Karp et al. 1990): Consider a graph $G(I, T, E)$ where the vertices in $I$, called resources (may correspond to advertisers in Internet advertising and tasks in Crowdsourcing), are known in advance. Vertices in $T$, called arrivals (corresponding to customers, ad slots, workers), are sequentially revealed one at a time. When a vertex $t \in T$ arrives, the set of edges $(i, t) \in E$ is revealed. Without loss of generality (w.l.o.g.), the vertices in $T$ arrive in the order of their index. After each arrival, the (online) algorithm must make an irrevocable decision to offer at most one (available/unmatched) vertex $i$ with an edge to $t$. Unmatched arrivals depart immediately and cannot be matched later. The goal is to maximize the total number of matches and the performance of online algorithm is compared against an optimal offline algorithm that knows the entire graph $G$ in advance. Formally, let $O P T(G)$ denote the optimal achievable by an offline algorithm and $A(G)$ the expected value achieved by a (possibly randomized) online algorithm $A$. The goal is to design an algorithm that maximizes the competitive ratio,

$$
\min _{G} \frac{A(G)}{O P T(G)}
$$

Karp et al. (1990) proposed and analyzed the Ranking algorithm that orders all offline vertices in a random permutation and matches every online vertex to the highest ranked neighbor available. They showed that Ranking attains the best possible competitive ratio of $(1-1 / e)$ for online bipartite matching (see Birnbaum and Mathieu (2008), Goel and Mehta (2008), Devanur et al. (2013) for simplified analysis).

In many applications, matches may fail with non-zero probability and the success or failure of a match is only revealed after the match has been made. This post-allocation stochasticity arises, for instance, in Internet advertising where with some probability a user may not click on the ads shown. In the popular pay-per-click model, an advertiser pays only if the user actually clicks i.e., the match succeeds. Similarly, in personalized recommendations new revenue is generated only if the customer buys an offered product.

Online matching with stochastic rewards: Motivated by the observations above, Mehta and Panigrahi (2012) introduced a generalization of online bipartite matching where the objective is to maximize the expected number of successful matches. Each edge $(i, t)$ has a success probability $p_{i t}$ that is revealed online along with the edge. When an algorithm makes a match, the arrival accepts (successful match or reward) with probability given by the corresponding edge. The success of a given match is independent of past ones and the outcome of the stochastic reward is revealed only after the match is made. An unsuccessfully matched arrival departs immediately, leaving 
the resource available for future re-match. A natural generalization of this problem allows vertexweighted rewards where each resource $i \in I$ has a (possibly different) reward $r_{i}{ }^{1}$. This is the setting we study.

The introduction of stochastic rewards raises an interesting question regarding the nature of the offline benchmark to compare against online algorithms. In addition to knowing all edges and their probabilities in advance, should the offline algorithm know the realizations of the rewards too? Unsurprisingly, such a benchmark turns out to be too strong for any meaningful bound ${ }^{2}$. A natural alternative is to compare against adaptive offline algorithms that know the arrivals, edges and edge probabilities in advance but must sequentially adapt to the outcomes of matches. In particular, rewards are realized only after matching decisions are made and offline can match any arrival at most once. Though offline knows all arrivals in advance, the sequence in which offline makes matching decisions gives rise to different benchmarks.

- Clairvoyant: An often used benchmark where the offline algorithm makes matching decisions in order of the arrival sequence i.e., vertex $t \in T$ is matched only after matching decisions for all vertices $t^{\prime}<t$ have been made (Golrezaei et al. 2014, Ma and Simchi-Levi 2018, Chan and Farias 2009, Gong et al. 2019, Feng et al. 2019).

- Fully Offline: Algorithm can adaptively choose an order for matching vertices in $T$, given the realized outcomes of previous matches (Grammel et al. 2021, Borodin et al. 2020).

Note that in the deterministic case (all probabilities are one) these benchmarks are identical since the order in which vertices are matched does not matter given knowledge of the entire arrival sequence. In general, there are merits to using either benchmark. On one hand, fully offline is a stronger benchmark than clairvoyant. On the other hand, clairvoyant benchmark generalizes quite naturally to other settings with post-allocation stochasticity. For instance, in case of reusable resources where successfully matched resources can be re-matched after some stochastic duration (Gong et al. 2019), allowing offline to match in an order different from the order of arrivals does not lead to a meaningful benchmark. Due to this generalizability, our main focus in this paper is to compare against clairvoyant benchmark. Interestingly, we find that our results hold more strongly against fully offline as well. For simplicity, we refer to clairvoyant and fully offline collectively as offline.

\footnotetext{
${ }^{1}$ If rewards are allowed to depend on both the arrival and resource the problem becomes much harder. It was shown in Aggarwal et al. (2011) that in this case no algorithm with competitive ratio better than $O\left(\frac{1}{|T|}\right)$ is possible.

${ }^{2}$ Consider a single arrival with an edge to $n \geq 1$ identical resources. Each edge has a probability of success $1 / n$. A benchmark that knows realizations of rewards attains expected reward at least $(1-1 / e)$. However, no online algorithm can achieve a reward better than $1 / n$.
} 
Unfortunately, the performance of offline can be hard to characterize exactly. However, observe that an upper bound on offline is easily given by the optimum of the following LP (see Ma and Simchi-Levi (2018), Golrezaei et al. (2014) for a formal proof).

$$
\begin{aligned}
O P T(L P)=\max & \sum_{(i, t) \in E} p_{i t} r_{i} x_{i t} \\
\text { s.t. } & \sum_{t \in T} p_{i t} x_{i t} \leq 1 \quad \forall i \in I \\
& \sum_{i \in I} x_{i t} \leq 1 \quad \forall t \in T \\
& 0 \leq x_{i t} \leq 1 \quad \forall(i, t) \in E,
\end{aligned}
$$

here $x_{i t}$ is a decision variable corresponding to the fraction of resource $i$ matched to arrival $t$. Constraints (1) enforce that the capacity constraint is satisfied in expectation, for every resource. We let the capacity of each resource be 1 w.l.o.g.. ${ }^{3}$ Similarly, constraints (2) enforce that the matched fractions of every arrival sum to at most 1 . We call this the expectation LP as the capacity constraints need only hold in expectation. Due to the simplicity of this LP, when evaluating competitive ratios against offline one often chooses to use the upper bound OPT $(L P)$.

Previous work: Golrezaei et al. (2014) and Mehta et al. (2015) showed that it is possible to obtain $1 / 2$ competitive guarantee against the expectation LP benchmark using deterministic greedy policies. Finding an algorithm that is provably better than naïve greedy is typically the main objective in this class of online allocation problems (Mehta et al. 2007, Mehta and Panigrahi 2012, Mehta et al. 2013). This has proven to be a major challenge for the stochastic rewards setting. Progress has been made in special cases with remarkable new insights. In applications such as online-advertisement, the edge probabilities or click-through-rates are sometimes quite small (Mehta et al. 2013). Consequently, this is a previously well studied case for the stochastic rewards problem. In particular, Mehta and Panigrahi (2012) found that for identical rewards and identical probabilities i.e., $r_{i}=1, \forall i \in I$ and $p_{i t}=p \forall(i, t) \in E$ : Assigning arrivals to available neighbors with the least number of failed matches in the past (called StochasticBalance) is 0.567 competitive as $p \rightarrow 0$. They also showed that Ranking is 0.534 approximate for arbitrary value of $p$. Complementing these lower bounds, they showed that no algorithm has a competitive ratio better than $0.621<$ $(1-1 / e)$ against the LP. This indicates that perhaps the problem is strictly harder than the classical

\footnotetext{
${ }^{3}$ Results for the case of unit capacity of each resource generalize to arbitrary capacities. Given a setting with inventory $c_{i}$ for resource $i$, consider a unit inventory setting where in place of resource $i$ we now have $c_{i}$ resources each with inventory of 1 and arrivals that have edges to all $c_{i}$ vertices for every edge $(i, t)$ in the original instance. Offline algorithm knows all arrivals in advance and thus knows these copies represent the same resource. Therefore, the optimal offline value remains unchanged and an algorithm for the unit inventory case can be used for arbitrary inventory levels without loss in guarantee.
} 
setting of Karp et al. (1990), where a $(1-1 / e)$ guarantee is possible. Mehta et al. (2015) studied the case of heterogeneous and vanishingly small edge probabilities and gave a 0.534 competitive algorithm (called SemiAdaptive). No results beating 1/2 are known to us for other cases, or when the rewards $r_{i}$ are not identical, even for identical probabilities.

\subsection{Our Results}

Our first result establishes the best possible guarantee of $(1-1 / e)$ for a special case of online matching with stochastic rewards where every edge probability is decomposable i.e., is a product of two terms corresponding to each of the two vertices ${ }^{4}$.

Theorem 1. For decomposable probabilities $p_{i t}=p_{i} p_{t} \forall(i, t) \in E$, Algorithm 1 is $(1-1 / e)$ competitive against offline (both clairvoyant and fully offline). This is the best possible competitive ratio guarantee.

Algorithm 1 computes a randomly perturbed per-unit reward for every resource and makes each match with the goal of maximizing the expected perturbed reward from the match. The algorithm is formally presented in Section 3. Observe that the case of decomposable probabilities (with otherwise arbitrary magnitudes) includes the case of identical probabilities $p_{i t}=p \forall(i, t) \in E$, for which Mehta and Panigrahi (2012) showed that there is no $0.621<(1-1 / e)$ competitive algorithm when comparing with $O P T(L P)$. Theorem 1 establishes that this upper bound is largely due to the limitations of the expectation LP benchmark (see Section 3.1). To obtain a tighter bound on offline we introduce a novel and intuitive path based program. In contrast to the expectation LP, this program has a constraint for every possible sample path instance of the stochastic reward process, along with constraints that enforce the independence of offline's decisions from the outcome of stochastic reward. This allows us to achieve the best possible guarantee of $(1-1 / e)$ with a natural generalization of an algorithm from deterministic online matching.

Let $p=\max _{(i, t) \in E} p_{i t}$, denote the maximum edge probability in a given instance. For general (not necessarily) decomposable probabilities, we establish the following guarantee.

TheOrem 2. There exists a constant $\eta>0$ such that Algorithm 2 is $(1-\eta \sqrt{p}) 0.596$ competitive against offline for $p \leq 0.38$.

Similar to Algorithm 1, Algorithm 2 matches each arrival greedily based on perturbed rewards. However, the perturbations are deterministic and change over time. The algorithm is formally presented in Section 4. Observe that, for instances with vanishingly small edge probabilities i.e., $p \rightarrow 0$,

\footnotetext{
${ }^{4}$ Note that similar special cases have been considered in other online matching settings. For instance, Charikar et al. (2014) consider a deterministic online matching problem where each edge has a weight $w_{i t}$ and the goal is find a matching with maximum total weight. They consider the special case where $w_{i t}=w_{i} \times w_{t}, \forall(i, t) \in E$, and give a $(1-1 / e)$ competitive algorithm for this setting.
} 


\begin{tabular}{|l|l|l|l|}
\cline { 3 - 3 } \multicolumn{1}{c|}{} & \multirow{2}{*}{ Identical rewards } & \multicolumn{2}{|c|}{ Vertex-weighted rewards } \\
\cline { 3 - 3 } \multicolumn{1}{c|}{} & & $c_{i}=1$ & $c_{i} \rightarrow \infty$ \\
\hline$p_{i t}=p \rightarrow 0$ & $0.567 \rightarrow(1-1 / \boldsymbol{e})$ & \multirow{2}{*}{$0.5 \rightarrow(1-1 / \boldsymbol{e})$} & \\
& \multirow{2}{*}{$(1-1 / e)$} \\
\hline$p_{i t}=p_{i} p_{t}$ & $0.500 \rightarrow(1-1 / \boldsymbol{e})$ & & \\
\hline$p_{i t} \rightarrow 0$ & $0.534 \rightarrow \mathbf{0 . 5 9 6}$ & $0.5 \rightarrow \mathbf{0 . 5 9 6}$ & \\
\hline General $p_{i t}$ & $1 / 2$ & $1 / 2$ & \\
\hline
\end{tabular}

Table 1 Summary of best known competitive ratio guarantees in relevant settings. Here $c_{i}$ represents the number of copies of each vertex $i \in I$ available, with $c_{i}=1$ being the most general case. Entries in bold represent our contributions.

the theorem implies a guarantee of 0.596. Recall that the importance of the special case of small probabilities stems from the observation that in applications such as online-advertisement, the probabilities or click-through-rates are often quite small (Mehta et al. 2013). The path based program that we introduce to prove Theorem 1 appears to be insufficient for analyzing non-decomposable probabilities even when they are vanishingly small. To show Theorem 2, we develop a novel LP free analysis technique that bypasses the need for an LP based upper bound and uses path-based arguments to directly compare online algorithms with offline.

A well studied regime for online resource allocation problems, including the stochastic rewards setting, is when we have a large number of copies $c_{i}$ for every vertex $i \in I$. In this large capacity regime, previous results show that one can achieve the best possible $(1-1 / e)$ guarantee against expectation LP (Golrezaei et al. 2014, Mehta et al. 2007). Why does the expectation LP benchmark admit this guarantee only for large inventory? To answer this question, in Section 5 we show that when $c_{i} \rightarrow+\infty \forall i \in I$, the expectation LP and offline benchmark converge to the same optimal value.

\subsection{Related Work}

We briefly review the vast body of literature on online resource allocation, including work we discussed previously to put things into perspective. Karp et al. (1990) introduced the online bipartite matching problem and proposed the optimal $(1-1 / e)$ competitive Ranking algorithm. Birnbaum and Mathieu (2008), Goel and Mehta (2008) considerably simplified (and completed) the original analysis. Subsequently, Aggarwal et al. (2011), proposed the Perturbed-Greedy algorithm and showed that it is $(1-1 / e)$ competitive more generally for the case of online vertex-weighted bipartite matching. Devanur et al. (2013) gave an elegant and intuitive randomized primal-dual framework for proving these results. Their framework applies to and simplifies several other settings, such the related AdWords problem of Mehta et al. (2007) (and the earlier result for $b$-matchings by Kalyanasundaram and Pruhs (2000)). There have also been a series of results on different types of stochastic arrival models, where there is distributional information in the arrivals that can be 
exploited for better results (for example, Devanur and Hayes (2009)), as well as simultaneous guarantees in adversarial and stochastic arrival settings (for instance, Mirrokni et al. (2012)). For a detailed survey of these models and results we refer the reader to a monograph by Mehta et al. (2013).

Moving on to settings with stochastic rewards, Mehta and Panigrahi (2012) introduced the model we consider here and focused on the case where all rewards and edge probabilities are identical. They showed the first results beating $1 / 2$ (and achieving 0.534 ) for this setting and motivated the case of small edge probabilities. Golrezaei et al. (2014), considered a broad generalization of stochastic rewards where one offers a set or an assortment of resources to each arrival. The arrival then chooses at most one resource from this set based on a choice model that is revealed online at the time of arrival. With the objective of maximizing total expected reward, they showed that when the number of copies (inventory) of each resource approaches $+\infty$, an inventory balancing algorithm $^{5}$ is asymptotically $(1-1 / e)$ competitive. For the most general case of unit inventory, their algorithm reduces to the Greedy algorithm and is $1 / 2$ competitive. Of course, this also establishes a $1 / 2$ competitive result for stochastic rewards with arbitrary vertex-weights and edge probabilities. The $1 / 2$ competitiveness of a non-adaptive Greedy algorithm for identical rewards was also shown independently by Mehta et al. (2015). They also gave a 0.534 competitive algorithm for identical rewards and heterogeneous but vanishingly small edge probabilities. Note that in contrast to Mehta and Panigrahi (2012) and Mehta et al. (2015), Golrezaei et al. (2014) evaluate competitiveness against the clairvoyant benchmark. However, for the sake of analysis they resort to an upper bound on the performance of clairvoyant and use the expectation LP. Clairvoyant algorithms are a commonly used benchmark in various other settings that include the element of stochastic rewards and more generally, other forms of post-allocation stochasticity (for instance, see Ma and SimchiLevi (2018), Chan and Farias (2009), Gong et al. (2019), Goyal et al. (2021)).

Since the online/arXiv version of this paper first appeared, there have been further developments of interest. In concurrent work, Grammel et al. (2021) consider at a setting where for each arrival a finite number of rematches are allowed in case of unsuccessful matches. They observe that in this case the problem for a single arrival is optimally solved by a dynamic program (DP). Using this insight show that the DP based greedy algorithm is 0.5 competitive. Further, they highlight several limitations of the LP benchmark and advocate the use of fully offline as a more natural benchmark for future work. Subsequently, Huang and Zhang (2020) gave a 0.572 competitive for heterogeneous and strongly vanishing probabilities $(p=1 / f(n)$, where $f(n)$ is a strictly increasing function of the number of resources $n$ ). Their result is shown against the expectation LP benchmark. Thus,

${ }^{5}$ A generalization of the algorithm for AdWords in Mehta et al. (2007) 
while their benchmark is stronger than offline the stronger assumption on smallness of probabilities and different numerical guarantee (0.572 in Huang and Zhang (2020) vs. 1-1/e and 0.596 here), renders their results incomparable with ours. Very recently, Udwani (2021) analyzed a variant of Algorithm 1 for an online allocation setting with unknown resource capacities. A byproduct of his result is that Algorithm 1 is at least 0.522 competitive for stochastic rewards with vanishing edge probabilities.

There is also a stream of work that looks at the aspect of stochastic rewards with stochastic arrivals and other related (including offline) versions of the problem. In particular, Bansal et al. (2010) were the first to study stochastic rewards in the context of bipartite matching. They considered an offline probe-commit version of the problem where all edges and probabilities are known in advance and one must probe edges with the restriction that if a probed edge exists, it must be included in the matching. We refer the reader to Grammel et al. (2021) and Borodin et al. (2020) for a more detailed review of these settings.

Outline for rest of the paper: We start by introducing notation in Section 2. In Section 3, we consider the case of decomposable probabilities and present Algorithm 1. Section 3.1 discusses the challenges with using the randomized primal-dual framework of Devanur et al. (2013) for the stochastic rewards problem. This motivates the consideration of our path based analysis in Section 3.2 and Section 3.3. In Section 4, we consider the case of small (not necessarily decomposable) probabilities. We start by presenting Algorithm 2, followed by the proof of Theorem 2 (Section 4.1 to Section 4.4). Section 5 completes the picture with a convergence result in the large inventory regime. Finally, in Section 6 we conclude with a review of some relevant open problems.

\section{Preliminaries}

Recall the problem definition. We have a bipartite graph $G$ with a set $I$ of $n$ offline vertices, also called resources. An arbitrary number of vertices $t \in T$ arrive online. We use the index of arrivals $t$ to also denote their order in time. So assume vertex $t \in T$ arrives at time $t$. All edges $(i, t)$ incident on vertex $t$ are revealed when $t$ arrives, along with a corresponding probability of success

$p_{i t}$. On each new arrival, we must make an irrevocable decision to match the arrival to any one of the available offline neighbors. Once a match, say $(i, t)$, is made, it succeeds with probability $p_{i t}$ independent of all other events. If the match succeeds, resource $i$ unavailable to future arrivals and leads to a reward $r_{i}$. The objective is to maximize the expected reward summed over all arrivals. Throughout the paper, we use ALG to denote the online algorithm under consideration as well its expected total reward. Similarly, OPT denotes the (fully) offline benchmark and its expected total reward. W.l.o.g., let OPT be a deterministic algorithm. 
Lemma 1. The optimal fully offline and clairvoyant algorithms are deterministic and given by a (possibly exponential time) dynamic program.

We provide a proof in Appendix A.2. While the ideas in this paper can be used for comparison with randomized benchmark algorithms, the fact that OPT is deterministic simplifies presentation of the key ideas.

For edge $(i, t) \in E$, let $\mathbb{1}(i, t)$ be an indicator random variable that denotes the success/failure of the edge. So $\mathbb{1}(i, t)$ equals 1 w.p. $p_{i t}$. Recall that, the success/failure of an edge is independent of the outcome of other edges. Let $\omega$ denote a sample path over the success/failure of all edges. Formally, $\omega$ defines for every edge $(i, t) \in E$, the value $\mathbb{1}(i, t)=\mathbb{1}^{\omega}(i, t)$ for the success/failure of $(i, t)$. Conditioned on $\omega$, any given (online or offline) algorithm observes the outcome $\mathbb{1}^{\omega}(i, t)$ after matching $i$ to $t$. Outcomes for edges not included in the matching are not revealed to the algorithm. Let $\Omega$ denote the universe of all sample paths $\omega$. For simplicity, we use $E_{\omega}[\cdot]$ to denote expectation over the randomness in outcomes of all edges. We use $\omega_{-i t}$ to represent part of sample path $\omega$ that defines the success/failure of all edges except edge $(i, t) \in E$. In other words, $\omega_{-i t}$ determines values of random variables $\mathbb{1}\left(j, t^{\prime}\right)$, for all edges $\left(j, t^{\prime}\right) \neq(i, t)$. Overloading notation, we let $E_{\omega}\left[\cdot \mid \omega_{-i t}\right]$ denote expectation over randomness in outcomes of edge $(i, t)$, with the outcomes of all other edges fixed according to $\omega_{-i t}$. Let $E_{\omega_{-i t}}[\cdot]$ denote expectation over the randomness in outcomes of all edges except $(i, t)$.

\section{Decomposable Probabilities $\left(p_{i t}=p_{i} \cdot p_{t}\right)$}

Our first algorithm for online matching with stochastic rewards computes a randomly perturbed per-unit reward for every resource and makes each match with the goal of maximizing the expected perturbed reward from the match.

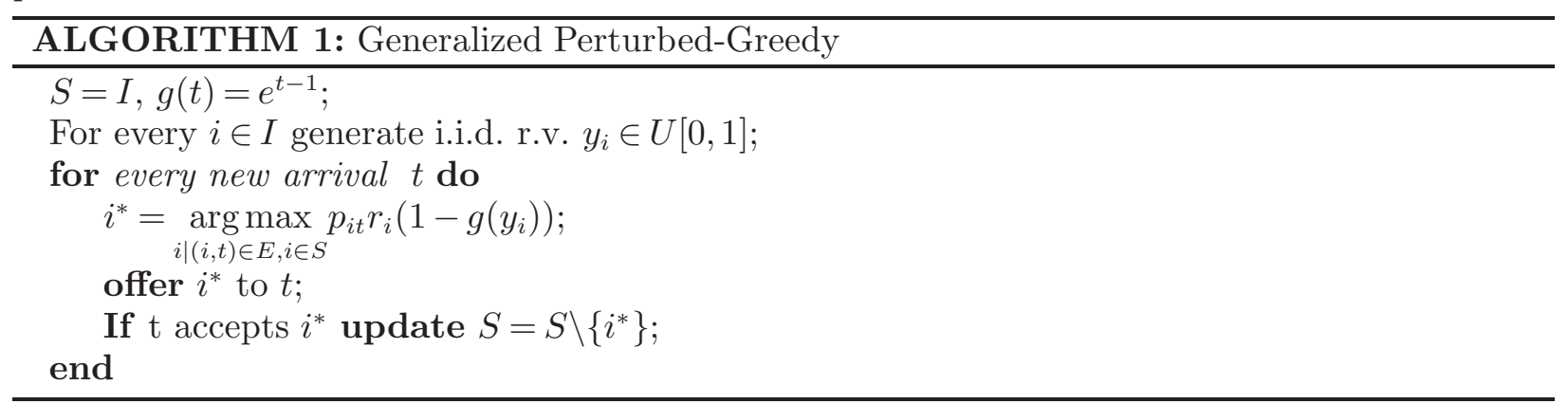

Algorithm 1 matches greedily w.r.t. expected reduced rewards $p_{i t} r_{i}\left(1-g\left(y_{i}\right)\right)$. We refer to random variable $y_{i}$ as the seed of resource $i$. The choice of scaling function $g(\cdot)$ naturally influences the performance of the algorithm. We let the analysis dictate this choice. As it turns out, $g(x)=e^{x-1}$ achieves the best possible performance guarantee of $(1-1 / e)$. 
When $p_{i t}=1 \forall(i, t) \in E$, Algorithm 1 is exactly the Perturbed-Greedy algorithm of Aggarwal et al. (2011). Devanur et al. (2013) show that Perturbed-Greedy is $(1-1 / e)$ competitive for deterministic online matching using the primal-dual approach. To analyze Algorithm 1 for stochastic rewards, we first explore a similar primal-dual analysis with the expectation LP benchmark. Since Algorithm 1 offers a natural extension of Perturbed-Greedy, at the outset it might even seem that the analysis in Devanur et al. (2013) may generalize directly. In the next section, we demonstrate the challenge with this approach through a simple example. Subsequently, we introduce a stronger benchmark, called the path based program (PBP), that we use to show a tight guarantee for Algorithm 1 against offline. Finally, in Section 3.4 we discuss (through an example) the challenges with extending the analysis to non-decomposable probabilities.

\subsection{Challenges with using Expectation LP Benchmark}

Let OPT denote the expected reward of offline. In absence of the stochastic component (all probabilities one), the offline and LP converge and we have OPT $=O P T(L P)$. In general, it is known that there are simple instances with a gap of up to $(1-1 / e)$ between OPT and $O P T(L P)$ i.e., $O P T(L P)$ can be as large as $(1-1 / e)^{-1} \mathrm{OPT}^{6}$ However, the gap between $O P T(L P)$ and OPT does not preclude the possibility of showing a strong competitive ratio guarantee against $O P T(L P)$. After all, a $1 / 2$ competitive result is known against the expectation LP benchmark in even more general settings (Golrezaei et al. 2014).

The LP based primal-dual framework of Buchbinder et al. (2007) and Devanur et al. (2013) is, arguably, the most versatile and general technique for proving guarantees for online matching and related problems. For the stochastic rewards problem, there are fundamental obstacles to using this technique with the expectation LP as a benchmark, and previous work explores novel approaches instead (Mehta and Panigrahi 2012, Mehta et al. 2015). Let us understand one such hurdle from the context of the framework in Devanur et al. (2013). We start with the dual of the expectation LP,

$$
\begin{array}{ll}
\min & \sum_{t \in T} \lambda_{t}+\sum_{i \in I} \theta_{i} \\
\text { s.t. } & \lambda_{t}+p_{i t} \theta_{i} \geq p_{i t} r_{i} \quad \forall(i, t) \in E \\
& \lambda_{t}, \theta_{i} \geq 0 \quad \forall t \in T, i \in I
\end{array}
$$

Primal-dual certificate (Devanur et al. 2013): To prove $\alpha$ competitiveness for an online algorithm with expected reward ALG, it suffices to find a set of non-negative values $\lambda_{t}, \theta_{i}$ such that,

${ }^{6}$ Consider one unit of a single resource with unit reward and $m$ identical arrivals that have an edge to the resource. Suppose that all edge probabilities are identically equal to $1 / \mathrm{m}$. The best that any offline algorithm can do is to match each arrival as long as the resource is still available. While this has expected reward $(1-1 / e)$ for $m \rightarrow+\infty$, the optimal value of LP equals 1 (Grammel et al. 2021, Mehta and Panigrahi 2012). 
(i) $\lambda_{t}+p_{i t} \theta_{i} \geq \alpha p_{i t} r_{i}, \quad \forall(i, t) \in E$,

(ii) $\mathrm{ALG} \geq \sum_{t \in T} \lambda_{t}+\sum_{i \in I} \theta_{i}$,

Now, consider Algorithm 1 and let ALG denote both the algorithm and its expected reward. Inspired by Devanur et al. (2013), we consider the following path based setting of dual variables:

Given a seed $Y=\left(y_{i}\right)_{i \in I}$ (used in Algorithm 1) and sample path $\omega$ over the edge outcomes, when the algorithm offers vertex $i$ to arrival $t$, set $\lambda_{t}^{\omega}\left(y_{i}\right)=r_{i}\left(1-g\left(y_{i}\right)\right) \mathbb{1}^{\omega}(i, t)$ and increase the value of $\theta_{i}^{\omega}\left(y_{i}\right)$ by $r_{i} g\left(y_{i}\right) \mathbb{1}^{\omega}(i, t)$. Let all unset variables be 0 .

It can be shown that the sum $\sum_{t} \lambda_{t}^{\omega}\left(y_{i}\right)+\sum_{i} \theta_{i}^{\omega}\left(y_{i}\right)$ equals the total reward of the algorithm on every sample path given by $Y, \omega$. Let $E_{\omega, Y}[\cdot]$ denote the expectation w.r.t. randomness in $Y$ as well as $\omega$. Define $\hat{\lambda}_{t}=E_{\omega, Y}\left[\lambda_{t}^{\omega}\left(y_{i}\right)\right]$ and $\hat{\theta}_{i}=E_{\omega, Y}\left[\theta_{i}^{\omega}\left(y_{i}\right)\right]$. Then, to prove $(1-1 / e)$ competitiveness of Algorithm 1, it suffices to show that that $\left\{\hat{\lambda}_{t}\right\}_{t \in T}$ and $\left\{\hat{\theta}_{i}\right\}_{i \in I}$ satisfy constraint (i) with $\alpha=(1-1 / e)$ for every edge $(i, t)$. When all edge probabilities are identically 1 , for $g(x)=e^{x-1}$, this follows directly from Lemma 2.4 in Devanur et al. (2013). Unfortunately, this is not true for non-unit probabilities. Consider the simplest possible example where the first arrival $t=1$ has an edge to resource $i$ and no other edges are incident on $i$ in the future. In this case, ALG always matches $i$ to $t$ and we have,

$$
\hat{\lambda}_{t}=p_{i t} r_{i} E_{y_{i}}\left[1-g\left(y_{i}\right)\right], \hat{\theta}_{i}=p_{i t} r_{i} E_{y_{i}}\left[g\left(y_{i}\right)\right] .
$$

Thus,

$$
\hat{\lambda}_{t}+p_{i t} \hat{\theta}_{i}=p_{i t} r_{i} E_{y_{i}}\left[1-g\left(y_{i}\right)\right]+p_{i t}^{2} r_{i} E_{y_{i}}\left[g\left(y_{i}\right)\right]
$$

For small $p_{i t}$, any term with $p_{i t}^{2}$ can be ignored and therefore, the expectation approaches $p_{i t} r_{i} E_{y_{i}}\left[\left(1-g\left(y_{i}\right)\right)\right]$. Consequently, for the natural trade-off function $g(x)=e^{x-1}$, we have, $\hat{\lambda}_{t}+\hat{\theta}_{i} \leq$ $\frac{1}{e} p_{i t} r_{i}$.

The main problem stems from fact that the LP only imposes capacity constraints in expectation, giving rise to a $p_{i t}^{2}$ term as we see above. One may consider other ways of setting dual values, such as the one in Buchbinder et al. (2007) or Golrezaei et al. (2014), however the resulting analysis faces similar issues. Our path based analysis circumvents this problem by imposing path-based dual constraints.

\subsection{Path Based Program (PBP)}

We start by constructing a tighter upper bound on offline via a path based formulation. Since OPT is deterministic (Lemma 1 ), we consider binary decision variables $x_{i t}^{\omega} \in\{0,1\} \forall(i, t) \in E, \omega \in \Omega$. On a given sample path $\omega$, let $x_{i t}^{\omega}$ equal 1 if offline matches $i$ to $t$, and 0 otherwise. Then, the following must be satisfied for every $\omega$,

$$
\sum_{t \mid(i, t) \in E} x_{i t}^{\omega} \mathbb{1}^{\omega}(i, t) \leq 1 \quad \forall i \in I
$$




$$
\sum_{i \mid(i, t) \in E} x_{i t}^{\omega} \leq 1 \quad \forall t \in T
$$

Constraints (4) capture the fact that any resource $i$ is used at most once on $\omega$. This is in contrast to the LP earlier, where this condition was imposed only in expectation over all sample paths. Similarly, constraints (5) capture that $t$ is matched to at most one vertex on every sample path. Recall that, offline does not know the outcome of an edge before it is chosen to be in the matching. Thus, the matching decision for arrival $t$ must not depend on the value of $\mathbb{1}(i, t)$ but may depend on the value of $\mathbb{1}\left(j, t^{\prime}\right)$ for $t^{\prime} \neq t$. We capture this structure with the constraints,

$$
x_{i t}^{\omega}=x_{i t}^{\omega^{\prime}} \quad \forall \omega, \omega^{\prime} \in \Omega \text { such that } \omega_{-i t}=\omega_{-i t}^{\prime} .
$$

Using (4), (5) and (6), we now formulate a path based LP relaxation with the objective of maximizing the total expected reward.

$$
\begin{array}{ll}
\text { PBP : } \max & E_{\omega}\left[\sum_{(i, t) \in E} r_{i} x_{i t}^{\omega} \mathbb{1}^{\omega}(i, t)\right] \\
\text { s.t. } & \sum_{t \mid(i, t) \in E} x_{i t}^{\omega} \mathbb{1}^{\omega}(i, t) \leq 1 \quad \forall i \in I, \omega \in \Omega \\
& \sum_{i \mid(i, t) \in E} x_{i t}^{\omega} \leq 1 \quad \forall t \in T, \omega \in \Omega \\
& x_{i t}^{\omega}=x_{i t}^{\omega^{\prime}} \quad \forall(i, t) \in E,\left\{\omega, \omega^{\prime} \in \Omega \mid \omega_{-i t}=\omega_{-i t}^{\prime}\right\} \\
& 0 \leq x_{i t}^{\omega} \leq 1 \quad \forall(i, t) \in E, \omega \in \Omega .
\end{array}
$$

Since offline must satisfy all constraints in the program, the values $x_{i t}^{\omega}$ generated by executing offline over sample paths $\omega \in \Omega$, yield a feasible solution for PBP. Therefore, the value of PBP offers an upper bound on the performance of the offline benchmark (both clairvoyant and fully offline).

The natural next step in developing a primal-dual argument is to construct a feasible solution for the dual of PBP. The dual now has exponentially many variables and constraints. Instead of dealing with those directly, the following lemma establishes a weak duality result with a simpler set of constraints i.e., finding a feasible solution for these constraints is easier and more natural.

Lemma 2. Consider non-negative variables $\lambda_{t}^{\omega}, \theta_{i}^{\omega}$ such that the following inequality holds for every edge $(i, t)$, partial sample path $\omega_{-i t}$, and some constant $\alpha>0$,

$$
E_{\omega}\left[\lambda_{t}^{\omega}+\theta_{i}^{\omega} \mathbb{1}^{\omega}(i, t) \mid \omega_{-i t}\right] \geq \alpha p_{i t} r_{i} .
$$

Then, for every feasible solution $\left\{x_{i t}^{\omega}\right\}$ of $P B P$,

$$
\frac{1}{\alpha} E_{\omega}\left[\sum_{t \in T} \lambda_{t}^{\omega}+\sum_{i \in I} \theta_{i}^{\omega}\right] \geq \sum_{(i, t) \in E} p_{i t} r_{i} E_{\omega}\left[x_{i t}^{\omega}\right] .
$$

Therefore, $E_{\omega}\left[\sum_{t \in T} \lambda_{t}^{\omega}+\sum_{i \in I} \theta_{i}^{\omega}\right] \geq \alpha O P T(P B P)$. 
Proof. Fix an arbitrary feasible solution $\left\{x_{i t}^{\omega}\right\}$, then for any given sample path $\omega$ we have,

$$
\sum_{t \in T} \lambda_{t}^{\omega} \sum_{i \mid(i, t) \in E} x_{i t}^{\omega}+\sum_{i \in I} \theta_{i}^{\omega} \sum_{t \mid(i, t) \in E} x_{i t}^{\omega} \mathbb{1}^{\omega}(i, t) \leq \sum_{t \in T} \lambda_{t}^{\omega}+\sum_{i \in I} \theta_{i}^{\omega}
$$

here we multiplied inequality (4) for each $i$ by $\theta_{i}^{\omega} \geq 0$, inequality (5) for each $t$ by $\lambda_{t}^{\omega} \geq 0$, and summed over all $i$ and every $t$. We re-write the inequality above as,

$$
\sum_{t \in T} \lambda_{t}^{\omega}+\sum_{i \in I} \theta_{i}^{\omega} \geq \sum_{(i, t) \in E} x_{i t}^{\omega} \lambda_{t}^{\omega}+\sum_{(i, t) \in E} x_{i t}^{\omega} \theta_{i}^{\omega} \mathbb{1}^{\omega}(i, t)
$$

Taking expectation (convex combination of linear constraints) over $\omega$,

$$
\begin{aligned}
E_{\omega}\left[\sum_{t \in T} \lambda_{t}^{\omega}+\sum_{i \in I} \theta_{i}^{\omega}\right] & \geq E_{\omega}\left[\sum_{(i, t) \in E} x_{i t}^{\omega}\left(\lambda_{t}^{\omega}+\theta_{i}^{\omega} \mathbb{1}^{\omega}(i, t)\right)\right] \\
& =\sum_{(i, t) \in E} E_{\omega_{-i t}}\left[E_{\omega}\left[x_{i t}^{\omega}\left(\lambda_{t}^{\omega}+\theta_{i}^{\omega} \mathbb{1}^{\omega}(i, t)\right) \mid \omega_{-i t}\right]\right] \\
& =\sum_{(i, t) \in E} E_{\omega_{-i t}}\left[x_{i t}^{\omega} E_{\omega}\left[\left(\lambda_{t}^{\omega}+\theta_{i}^{\omega} \mathbb{1}^{\omega}(i, t)\right) \mid \omega_{-i t}\right]\right] \\
& \geq \alpha p_{i t} r_{i} \sum_{(i, t) \in E} E_{\omega}\left[x_{i t}^{\omega}\right],
\end{aligned}
$$

here the first equation follows from the tower rule of expectation. The second equation follows from the fact that conditioned on $\omega_{-i t}, x_{i t}^{\omega}$ is deterministic (condition (6)). The final inequality follows from, $E_{\omega}\left[\lambda_{t}^{\omega}+\theta_{i}^{\omega} \mathbb{1}^{\omega}(i, t) \mid \omega_{-i t}\right] \geq \alpha p_{i t} r_{i}$. To finish the proof consider the objective of PBP.

$$
\begin{aligned}
O P T(\mathrm{PBP})=E_{\omega}\left[\sum_{(i, t) \in E} r_{i} x_{i t}^{\omega} \mathbb{1}^{\omega}(i, t)\right] & =\sum_{(i, t) \in E} E_{\omega_{-i t}}\left[E_{\omega}\left[r_{i} x_{i t}^{\omega} \mathbb{1}^{\omega}(i, t) \mid \omega_{-i t}\right]\right] \\
& =\sum_{(i, t) \in E} E_{\omega_{-i t}}\left[r_{i} x_{i t}^{\omega} E_{\omega}\left[\mathbb{1}^{\omega}(i, t) \mid \omega_{-i t}\right]\right] \\
& =\sum_{(i, t) \in E} E_{\omega_{-i t}}\left[p_{i t} r_{i} x_{i t}^{\omega}\right]=\sum_{(i, t) \in E} p_{i t} r_{i} E_{\omega}\left[x_{i t}^{\omega}\right]
\end{aligned}
$$

here the first equation follows form the tower rule of expectation, the second from condition (6), the third equation follows from the independence of $\mathbb{1}(i, t)$ from $\omega_{-i t}$ and the final equation also from condition (6).

\subsection{Path Based Dual Fitting}

Let ALG denote Algorithm 1 as well as its total expected reward. Armed with Lemma 2, we now focus on finding 'dual' values $\lambda_{t}^{\omega} \geq 0$ and $\theta_{i t}^{\omega} \geq 0$ such that,

$$
\begin{aligned}
E_{\omega}\left[\sum_{t \in T} \lambda_{t}^{\omega}+\sum_{i \in I} \theta_{i}^{\omega}\right] & =\mathrm{ALG}, \\
E_{\omega}\left[\lambda_{t}^{\omega}+\theta_{i}^{\omega} \mathbb{1}^{\omega}(i, t) \mid \omega_{-i t}\right] & \geq \alpha p_{i t} r_{i} \quad \forall(i, t) \in E, \omega_{-i t} \in \Omega .
\end{aligned}
$$


If we can find dual values that satisfy the system given by these linear constraints, then using Lemma 2 we that ALG is $\alpha$ competitive against offline. We start by defining a candidate solution for the system based on the decisions of Algorithm 1, followed by a proof of feasibility. This process is called dual fitting (Mehta et al. 2013).

Given the fact that we have external randomness in ALG due to seeds $\boldsymbol{Y}=\left(y_{i}\right)_{i \in I}$, we first define variables $\lambda_{t}^{\omega, Y}, \theta_{i}^{\omega, Y}$ and then set $\lambda_{t}^{\omega}=E_{Y}\left[\lambda_{t}^{\omega, Y}\right]$ and $\theta_{i}^{\omega}=E_{Y}\left[\theta_{i}^{\omega, Y}\right]$. To that end, we couple the stochastic rewards in ALG and PBP as follows.

Coupling between ALG and PBP: Given sample path $\omega \in \Omega$ we consider concurrent execution of ALG and PBP with match successes/failures given by $\omega$ i.e., both ALG and PBP see the same values $\mathbb{1}^{\omega}(i, t), \forall(i, t) \in E$.

Now, consider a path based generalization of the dual setting in Devanur et al. (2013). Initialize all dual variables to 0 and for any match $(i, t)$ in ALG set,

$$
\lambda_{t}^{\omega, Y}=r_{i}\left(1-g\left(y_{i}\right)\right) \mathbb{1}^{\omega}(i, t) \text { and increment } \theta_{i}^{\omega, Y} \text { by } r_{i} g\left(y_{i}\right) \mathbb{1}^{\omega}(i, t) .
$$

Clearly, $\lambda_{t}^{\omega, Y}$ is set uniquely since ALG offers at most one resource $i$ to arrival $t$. Also, $\theta_{i}^{\omega, Y}$ takes a non-zero value only if it is also accepted by some $t$ and if this occurs, $\theta_{i}^{\omega, Y}$ is never re-set. We have the following as an immediate consequence of this setting of dual values.

LEMma 3. For the candidate solution given by (9), equality (7) is satisfied.

Proof. Fix sample path $\omega$ and seed $Y$. Let $M^{\omega, Y}$ denote the matching output by ALG on the resulting sample path. By definition of the dual values in (9), we have that $\sum_{t} \lambda_{t}^{\omega, Y}+\sum_{i} \theta_{i}^{\omega, Y}$ is exactly $\sum_{(i, t) \in M^{\omega, Y}} \mathbb{1}^{\omega}(i, t) r_{i}$. Taking expectation gives the desired.

It remains to show (8). Before we proceed, let us reflect back on the issue highlighted in Section 3.1 and see how the path based approach resolves it. Consider a simple instance where the first arrival, $t=1$, has an edge to resource $i$ and no other arrival is adjacent to $i$. ALG matches $i$ to $t$ and we have,

$$
\begin{aligned}
E_{\omega, Y}\left[\lambda_{t}^{\omega, Y}+\theta_{i}^{\omega, Y} \mathbb{1}^{\omega}(i, t)\right] & =r_{i} E_{\omega, Y}\left[\left(1-g\left(y_{i}\right)\right) \mathbb{1}^{\omega}(i, t)+g\left(y_{i}\right)\left(\mathbb{1}^{\omega}(i, t)\right)^{2}\right] \\
& =r_{i} E_{\omega, Y}\left[\mathbb{1}^{\omega}(i, t)\right]=p_{i t} r_{i} .
\end{aligned}
$$

Clearly, we do not face the issue highlighted in Section 3.1. In the path based approach, we are able to correlate random variables $\theta_{i}^{\omega, Y}$ and $\mathbb{1}^{\omega}(i, t)$, and boost the expectation of the product $\theta_{i}^{\omega, Y} \mathbb{1}^{\omega}(i, t)$. In contrast, the dual feasibility condition coming out of the expectation LP has the term $p_{i t} \theta_{i}=E_{\omega}\left[\mathbb{1}^{\omega}(i, t)\right] E_{\omega, \boldsymbol{Y}}\left[\theta_{i}^{\omega, Y}\right]$, which does not allow for the possibility of correlating the two random variables.

We now show that inequalities (8) are satisfied with $\alpha=(1-1 / e)$, as long as the probabilities are decomposable. In fact, we show a stronger statement as described in the following lemma. 
Lemma 4. Consider an edge $(i, t) \in E$. Suppose that for the dual fitting given by (9), for every sample path $\omega_{-i t}$ and values $y_{j}$ for all $j \neq i$, denoted $Y_{-i}$, we have,

$$
E_{y_{i}}\left[E_{\omega}\left[\lambda_{t}^{\omega, Y}+\theta_{i}^{\omega, Y} \mathbb{1}^{\omega}(i, t) \mid \omega_{-i t}\right] \mid Y_{-i}\right] \geq(1-1 / e) p_{i t} r_{i}
$$

Then, inequality (8) is satisfied for edge $(i, t)$ with $\alpha=(1-1 / e)$.

Proof. Taking expectation over $Y_{-i}$ and using the tower rule of expectation we have, $E_{\omega}\left[E_{Y}\left[\lambda_{t}^{\omega, Y}+\theta_{i}^{\omega, Y} \mathbb{1}^{\omega}(i, t)\right] \mid \omega_{-i t}\right] \geq(1-1 / e) p_{i t} r_{i}, \forall \omega_{-i t}$. Taking expectation over $\omega_{-i t}$ completes the proof.

For the following discussion, fix an arbitrary edge $(i, t)$ (not necessarily chosen in the matching), sample path $\omega_{-i t}$, and random seed $Y_{-i}$ for every $j \neq i$. For brevity, we omit $\omega_{-i t}$ and $Y_{-i}$ from notation and let,

$$
E_{y_{i}}\left[E_{\omega}\left[\lambda_{i}^{\omega, Y}\right]\right]:=E_{y_{i}}\left[E_{\omega}\left[\lambda_{t}^{\omega, Y} \mid \omega_{-i t}\right] \mid Y_{-i}\right]
$$

Similarly,

$$
E_{y_{i}}\left[E_{\omega}\left[\theta_{i}^{\omega, Y} \mathbb{1}^{\omega}(i, t)\right]\right]:=E_{y_{i}}\left[E_{\omega}\left[\theta_{i}^{\omega, Y} \mathbb{1}^{\omega}(i, t) \mid \omega_{-i t}\right] \mid Y_{-i}\right]
$$

To show (10), we separately lower bound $E_{y_{i}}\left[E_{\omega}\left[\lambda_{i}^{\omega, Y}\right]\right]$ (Lemma 5 ) and $E_{y_{i}}\left[E_{\omega}\left[\theta_{i}^{\omega, Y} \mathbb{1}^{\omega}(i, t)\right]\right]$ (Lemma 6). Combining these lower bounds gives us the desired. The lower bound on $E_{y_{i}}\left[E_{\omega}\left[\lambda_{i}^{\omega, Y}\right]\right]$ is similar to Lemma 2.3 (Monotonicity Lemma) in Devanur et al. (2013). At a high level, the lower bound on $E_{y_{i}}\left[E_{\omega}\left[\theta_{i}^{\omega, Y} \mathbb{1}^{\omega}(i, t)\right]\right]$ follows from a "dominance" property similar to Lemma 2.2 in Devanur et al. (2013). However, our analysis has an interesting subtlety (absent in the classical case) due to the fact that matches can be unsuccessful. This is also where we need the decomposability assumption for edge probabilities. We discuss this further in the proof of Lemma 6.

With $\omega_{-i t}$ and $Y_{-i}$ fixed, let $M_{-i}$ denote the matching generated by ALG up to (and including) arrival $t$, when it is executed with the reduced set of vertices $I \backslash i$. Unlike the case where all edge probabilities are 1 , here $M_{-i}$ may have one offline vertex matched multiple times though only one match could have actually succeeded. Let $I_{-i}^{t}$ denote the set of available neighbors of $t$, in this execution of ALG without vertex $i$. Define $y_{i}^{c}$ such that,

$$
p_{i t} r_{i}\left(1-g\left(y_{i}^{c}\right)\right)=\max _{j \in I_{-i}^{t}} p_{j t}\left(1-g\left(y_{j}\right)\right) .
$$

Set $y_{i}^{c}=1$ if the set $I_{-i}^{t}$ is empty, and $y_{i}^{c}=0$ if no such value exists. Due to the monotonicity of $g(t)=e^{t-1}$, we have a unique value of $y_{i}^{c}$.

Lemma 5. Given edge $(i, t)$, sample path $\omega_{-i t}$, seed $Y_{-i}$, we have $E_{\omega}\left[\lambda_{t}^{\omega, Y}\right] \geq p_{i t} r_{i}\left(1-g\left(y_{i}^{c}\right)\right)$ for every $y_{i} \in[0,1]$. Thus, $E_{y_{i}}\left[E_{\omega}\left[\lambda_{i}^{\omega, Y}\right]\right] \geq p_{i t} r_{i}\left(1-g\left(y_{i}^{c}\right)\right)$. 
Proof. Given edge $(i, t)$, sample path $\omega_{-i t}$, and random seed $Y_{-i}$, let $I_{y_{i}}^{t}$ denote the set of available neighbors at $t$ when ALG is executed with the full vertex set and seed value $y_{i}$. Observe that if,

$$
\max _{j \in I_{y_{i}}^{t}} p_{j t}\left(1-g\left(y_{j}\right)\right) \geq p_{i t}\left(1-g\left(y_{i}^{c}\right)\right) \quad \forall y_{i} \in[0,1],
$$

then we have the desired. To show this inequality, it suffices to prove that $I_{-i}^{t} \subseteq I_{y_{i}}^{t} \forall y_{i} \in[0,1]$. Let $M_{y_{i}}$ denote the matching generated by ALG up to (and including) $t$ with seed $y_{i}$. The proof follows from the following cases.

Case I: In $M_{y_{i}}, i$ is not matched to any arrival prior to $t$. Thus, matchings $M_{-i}$ and $M_{y_{i}}$ are identical prior to arrival $t$ and $I_{y_{i}}^{t}=I_{-i}^{t} \cup\{i\}$.

Case II: In $M_{y_{i}}, i$ is matched prior to $t$. Let $t^{\prime}$ denote the first arrival matched to $i$ in $M_{y_{i}}$. For the sake of contradiction, let $\tau \leq t$ be the first arrival such that there exists a resource $j \in I_{-i}^{\tau} \backslash I_{y_{i}}^{\tau}$. Clearly, $\tau>t^{\prime}$ since $M_{-i}$ and $M_{y_{i}}$ are identical prior to $t^{\prime}$. Now, let $t_{j}<\tau$ be the arrival successfully matched to $j$ in $M_{y_{i}}$. So, we have the arrivals in the following order $t^{\prime} \leq t_{j}<\tau \leq t$. Since $j \in I_{-i}^{\tau}$, we have, $j \in I_{-i}^{t_{j}}$. Further, $I_{-i}^{t_{j}} \subseteq I_{y_{i}}^{t_{j}}$. Thus, $j \in I_{y_{i}}^{t_{j}}$ and by definition of ALG, $j$ must be (successfully) matched to $t_{j}$ in $M_{-i}$, contradiction. Thus, $I_{-i}^{\tau} \subseteq I_{y_{i}}^{\tau}$ for every $\tau \leq t$.

Lemma 6. Given edge $(i, t)$, sample path $\omega_{-i t}$, seed $Y_{-i}$ and decomposable edge probabilities $p_{i t}=$ $p_{i} p_{t} \forall(i, t) \in E$, we have $E_{y_{i}}\left[E_{\omega}\left[\theta_{i}^{\omega, Y} \mathbb{1}^{\omega}(i, t)\right]\right] \geq p_{i t} r_{i} \int_{0}^{y_{i}^{c}} g(x) d x$.

Proof. Given edge $(i, t)$, sample path $\omega_{-i t}$, and random seed $Y_{-i}$, consider a value $y_{i} \in\left[0, y_{i}^{c}\right)$. There are two possibilities, either $i$ is successfully matched before $t$ and unavailable at $t$, or $i$ is available when $t$ arrives. We will show that in the latter scenario ALG matches $i$ to $t$ but first, let us see how this proves the lemma. When $i$ is unavailable at $t, \theta_{i}^{\omega, Y}=r_{i} g\left(y_{i}\right)$ since $i$ was successfully matched to an arrival preceding $t$. In case $i$ is available and thus matched to $t$ (by assumption), $\theta_{i}^{\omega, Y}=r_{i} g\left(y_{i}\right) \mathbb{1}^{\omega}(i, t)$. Therefore, in both cases $E_{\omega}\left[\theta_{i}^{\omega, Y} \mathbb{1}^{\omega}(i, t)\right]=p_{i t} r_{i} g\left(y_{i}\right)$. Since this holds for all values of $y_{i}<y_{i}^{c}$ we have the desired.

To finish the proof we need to show that, for $y_{i}<y_{i}^{c}, i$ is matched to $t$ if available. In the classical/deterministic case this follows directly from $r_{i}\left(1-g\left(y_{i}\right)\right) \geq r_{i}\left(1-g\left(y_{i}^{c}\right)\right)$. In our case, we still have $p_{i t} r_{i}\left(1-g\left(y_{i}\right)\right) \geq p_{i t} r_{i}\left(1-g\left(y_{i}^{c}\right)\right)$. However, for some small enough value of $y_{i}$, say $y_{0}, i$ may be unsuccessfully matched to some arrival $t^{\prime}$ preceding $t$. This could free up a resource $j(\neq i)$ that was successfully matched to $t^{\prime}$ for $y_{i}>y_{0}$. So for $y_{i} \leq y_{0}$, the freed up resource $j$ may be matched to $t$ instead of $i$, even if $i$ was available at $t$. In such a scenario it is not a priori clear if we still have the desired lower bound on $\theta_{i}^{Y}$. Observe that if $i$ is never matched/offered to any arrival preceding $t$ then such an issue does not arise and the claim follows as in the deterministic case. So the interesting case is when $i$ is matched (unsuccessfully) prior to $t$ for some value $y_{i}<y_{i}^{c}$ and 
consequently, $t$ is matched to some $j(\neq i)$ such that, $E\left[\lambda_{t}^{Y}\right]=p_{j t} r_{j}\left(1-g\left(y_{j}\right)\right)>p_{i t} r_{i}\left(1-g\left(y_{i}^{c}\right)\right)$. In this case, consider the graph given by the set difference between the matching $M_{y_{i}}$ generated by ALG with seed value $y_{i}$ and the matching $M_{-i}$ generated by ALG when resource $i$ is excluded. On this difference graph, there exists a unique alternating path that includes both $i$ and $t$. Since ALG matches every arrival to the resource with maximum expected reduced reward, moving along the alternating path starting with the edge incident on $i$ and applying the decomposition of probabilities, we have for every edge $\left(i^{\prime}, t^{\prime}\right)$ on the path, $p_{i} r_{i}\left(1-g\left(y_{i}\right)\right) \geq p_{i^{\prime}} r_{i^{\prime}}\left(1-g\left(y_{i^{\prime}}\right)\right)$ (though it may be that, $\left.p_{i t} r_{i}\left(1-g\left(y_{i}\right)\right)<p_{i^{\prime} t^{\prime}} r_{i^{\prime}}\left(1-g\left(y_{i^{\prime}}\right)\right)\right)$. In particular, $p_{i} r_{i}\left(1-g\left(y_{i}\right)\right) \geq p_{j} r_{j}\left(1-g\left(y_{j}\right)\right)$ and thus, $p_{i t} r_{i}\left(1-g\left(y_{i}\right)\right) \geq p_{j t} r_{j}\left(1-g\left(y_{j}\right)\right)$. Therefore, if $i$ is available on arrival of $t$, the algorithm matches $i$ to $t$ (since ties occur w.p. 0 ).

Proof of Theorem 1. The proof follows by combining Lemmas 2, 3, 5 and 6, and using the fact that $1-g(y)+\int_{o}^{y} g(x) d x=(1-1 / e) \forall x \in[0,1]$ when $g(x)=e^{x-1}$.

\subsection{Obstacle for Non-decomposable Probabilities}

To understand the effect of decomposable probabilities in Algorithm 1, note that the values $p_{i} r_{i}(1-$ $\left.g\left(y_{i}\right)\right)$ induce an ordering over the vertices $i \in I$ that is independent of arrivals. Therefore, given any two resources $i, j$, and an arrival $t$ with an edge to both, Perturbed-Greedy prefers to offer $i$ over $j$ whenever $p_{i} r_{i}\left(1-g\left(y_{i}\right)\right)>p_{j} r_{j}\left(1-g\left(y_{j}\right)\right)$. This fixed ordering/ranking is a key component in the proof of Lemma 6. For general probabilities, Algorithm 1 may prefer $j$ over $i$ for arrival $t$ but vice versa for another arrival $t^{\prime}$.

Let us see an example with non-decomposable probabilities where Lemma 6 fails. Consider a $3 \times 3$ bipartite graph with arrival $t_{1}$ arriving first, followed by $t_{2}$, and finally $t_{3}$. Consider resources $i, j, k$ with resource $i$ adjacent to all three arrivals, resource $j$ adjacent to arrivals $t_{2}$ and $t_{3}$ and finally, resource $k$ adjacent solely to arrival $t_{1}$. Let the rewards $r_{i}, r_{j}$, and $r_{k}$ be identical and equal 1. Consider probabilities $p_{i t_{1}}=p_{k t_{1}}, p_{i t_{2}}=p_{j t_{2}}$ and $p_{i t_{3}}<p_{j t_{3}}$. Note that such probabilities are not decomposable. Let us focus on the dual feasibility of constraint corresponding to edge $\left(i, t_{3}\right)$. So we fix $y_{j}, y_{k}$ with $y_{j}>y_{k}$ and consider the following sample path before $t_{3}$ arrives, $\mathbb{1}^{\omega}\left(k, t_{1}\right)=0, \mathbb{1}^{\omega}\left(i, t_{1}\right)=1$ and $\mathbb{1}^{\omega}\left(i, t_{2}\right)=0, \mathbb{1}^{\omega}\left(j, t_{2}\right)=1$. Observe that $y_{i}^{c}=1$ and consider $E\left[\lambda_{t_{3}}^{Y}\right]$ and $E\left[\theta_{i}^{Y} \mathbb{1}\left(i, t_{3}\right)\right]$ as $y_{i}$ varies.

For $y_{i} \geq y_{j}, k$ is offered to $t_{1}, j$ is offered to and accepted by $t_{2}$, and $i$ is offered to $t_{3}$. Therefore, $E\left[\theta_{i}^{Y} \mathbb{1}\left(i, t_{3}\right)\right]=p_{i t_{3}} r_{i} g\left(y_{i}\right)$ and $E\left[\lambda_{t_{3}}^{Y}\right]=p_{i t_{3}} r_{i}\left(1-g\left(y_{i}\right)\right)$.

For $y_{k}<y_{i}<y_{j}, i$ is offered to but not accepted by $t_{2}$ and suppose $p_{i t_{3}}$ is sufficiently smaller than $p_{j t_{3}}$ so $j$ is offered to $t_{3}$. Thus, $E\left[\lambda_{t_{3}}^{Y}\right]=p_{j t} r_{j}\left(1-g\left(y_{j}\right)\right)$, but $\theta_{i}^{Y}=0$ for $y_{k}<y_{i}<y_{j}$.

Finally, for $y_{i} \leq y_{k}, i$ is offered to and accepted by $t_{1}, j$ is offered to and accepted by $t_{2}$ and $t_{3}$ is unmatched with $\lambda_{t_{3}}^{Y}=0=p_{i t_{3}} r_{i}\left(1-g\left(y_{i}^{c}\right)\right)$ and $E\left[\theta_{i}^{Y} \mathbb{1}\left(i, t_{3}\right)\right]=p_{i t_{3}} r_{i} g\left(y_{i}\right)$. 
Combining the pieces we have, $E_{y_{i}}\left[E\left[\lambda_{t_{3}}^{Y}+\theta_{i}^{Y} \mathbb{1}\left(i, t_{3}\right)\right]\right]=p_{i t_{3}} r_{i}\left(1-y_{j}\right)+p_{j t_{3}} r_{j}\left(1-g\left(y_{j}\right)\right)\left(y_{j}-y_{k}\right)+$ $p_{i t_{3}} r_{i} \int_{0}^{y_{k}} g(x) d x$. Clearly, Lemma 6 does not hold and the previous expectation can be $<0.5 p_{i t_{3}} r_{i}$. More concretely, let $y_{j}=1-\epsilon$ and $p_{j t_{3}}, p_{i t_{3}}$ be such that $p_{j t_{3}}\left(1-g\left(y_{j}\right)\right)=p_{i t_{3}}\left(1-g\left(y_{k}\right)\right)$. Then for $g(x)=e^{x-1}$,

$$
\begin{aligned}
E_{y_{i}}\left[E\left[\lambda_{t_{3}}^{Y}+\theta_{i}^{Y} \mathbb{1}\left(i, t_{3}\right)\right]\right] & =c \epsilon+p_{i t_{3}} r_{i}\left(1-g\left(y_{k}\right)\right)\left(1-y_{k}\right)+p_{i t_{3}} r_{i} \int_{0}^{y_{k}} g(x) d x \\
& \geq c \epsilon+p_{i t_{3}} r_{i} \min _{y}\left([1-g(y)](1-y)+\int_{0}^{y} g(x) d x\right) \\
& =c \epsilon+0.44 p_{i t_{3}} r_{i},
\end{aligned}
$$

where $c$ is some non-negative real number and the inequality is tight for $y_{k}=0.5571$. This shows that the existing analysis does not lead to a $(1-1 / e)$ guarantee for general edge probabilities. Note that using our analysis for $g(x)=1 / 2$ (which corresponds to the greedy algorithm) we get a guarantee of $1 / 2$ for general probabilities.

\section{Vanishing Probabilities $\left(p_{i t} \rightarrow 0\right)$}

In this section, we consider the case of vanishingly small heterogeneous probabilities. As demonstrated in the previous section, the path based primal-dual analysis does not yield a guarantee better than 0.5 in this setting. We overcome these challenges by introducing a new algorithm and a novel LP free analysis. Our algorithm is deterministic, and this feature eases some of the analytical challenges. It is worth noting that for arbitrary edge probabilities (including the classic online bipartite matching problem), no deterministic online algorithm can have a guarantee strictly better than 0.5 (Karp et al. 1990).

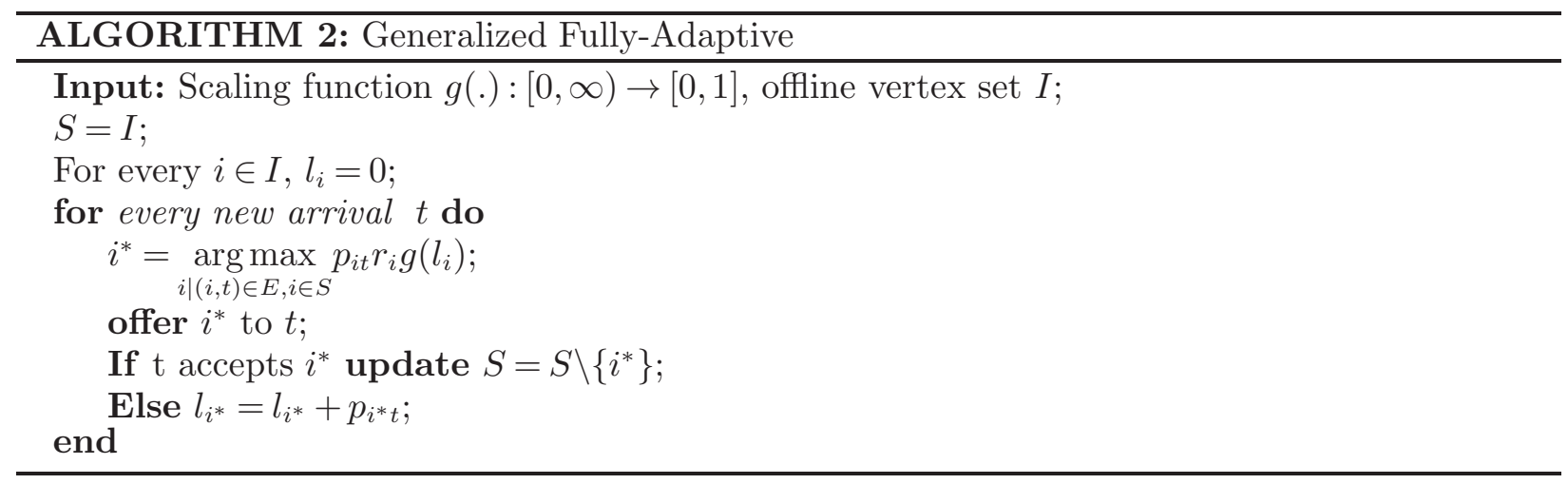

Observe that Algorithm 2 matches greedily w.r.t. expected reduced rewards $p_{i t} r_{i} g\left(l_{i}\right)$. Unlike Algorithm 1, the reduced rewards are now deterministic and change over time. Our algorithm generalizes the Fully-Adaptive algorithm proposed, but not analyzed, in Mehta and Panigrahi (2012) and Mehta et al. (2015). The choice of scaling function $g(\cdot)$ naturally influences the performance of the algorithm. At a high level, it is desirable to choose a decreasing function so that the reduced 
reward of a resource decreases every time it is matched. The guarantee of 0.596 stated in Theorem 2 is obtained with $g(t)=e^{t+1}\left(\int_{t+1}^{\infty} \frac{e^{-y}}{y} d y\right)$. We also consider other choices of $g(\cdot)$. In particular, we show that a simple inverse scaling function $g(t)=\frac{1}{\beta t+1}$, is at least 0.588 competitive. For the function $g(t)=e^{-\beta t}$, a natural generalization of the function $e^{-t}$ proposed in Mehta et al. (2015), we establish 0.581 competitiveness.

As we saw, our previous path based analysis is insufficient when probabilities do not decompose. In fact, the linear system given by (7) and (8) may not be feasible for $\alpha>0.5$. We resolve this difficulty via two new ideas. First, we introduce an alternative viewpoint of the reward process that allows a natural characterization of the probability of an algorithm successfully matching a given resource. This viewpoint generalizes an insight from Mehta and Panigrahi (2012), and is introduced in Section 4.1 and Section 4.2. Then, we convert the problem of finding a competitive ratio guarantee for Algorithm 2, to proving the feasibility of linear system. We introduce a new linear system that is a more relaxed (and easier to satisfy) than LP duality based linear systems. For instance, recall that the system given by (7) and (8) has a constraint for every edge in the graph (local constraints). In contrast, we now impose a single constraint for every resource (global constraints). The constraints are path-based and inspired by duality but do not necessarily correspond to the dual of a program. This makes the analysis LP free and, generally speaking, robust

to any issues related to tightness of a mathematical program. We introduce the LP free system in Section 4.3. Finally, in Section 4.4 we utilize these ingredients to prove Theorem 2.

\subsection{An Alternative Viewpoint}

Consider a deterministic algorithm $\mathcal{A}$ (online or offline) for the stochastic rewards problem. Let $\mathcal{A}$ be non-anticipative i.e., the outcome (success/failure) of every match is revealed to $\mathcal{A}$ only after the match is made and any vertex $t \in T$ can be matched at most once. Observe that, both Algorithm 2 and the offline benchmark are deterministic.

We interpret outcomes of matches as a sequential input to $\mathcal{A}$ i.e., every time $\mathcal{A}$ makes a match, an independent Bernoulli sample denoting success/failure of that match is provided to $\mathcal{A}$. Mehta and Panigrahi (2012) considered the case of identical edge probabilities $p$ and observed that instead of letting the success of each match be determined by an i.i.d. random variable, one could consider the following equivalent process. For each resource $i$, independently generate a starting budget $b_{i}$ from the distribution,

$$
\mathbb{P}\left(b_{i}=k p\right)=p(1-p)^{k-1}, \text { for } k \in\{1,2, \cdots\} .
$$

In executing $\mathcal{A}$, every match to $i$ reduces the remaining budget by $p$ and a match succeeds when the remaining budget reaches zero. So if the starting budget $b_{i}=k p$, the $k$-th match made to $i$ would 
succeed, and none before. Budgets $\left(b_{i}\right)_{i \in I}$ are sampled in advance but $\mathcal{A}$ is unaware of these values. Mehta and Panigrahi (2012) showed that budgets $\left(b_{i}\right)_{i \in I}$ define an alternative characterization of stochastic rewards i.e., given a matching, the probability that $\mathcal{A}$ outputs this matching is identical in both methods of generating random match outcomes.

For $p \rightarrow 0$, the distribution of budgets, (11), is well approximated by $\operatorname{Exp}(1)$, which denotes the exponential distribution with cumulative distribution $F(x)=1-e^{-x}$ for $x \geq 0$. To analyze Algorithm 2, we generalize this viewpoint (and approximation) to arbitrary probabilities.

Generalized Alternative Viewpoint: Let $U[0,1]$ denote the uniform distribution on interval $[0,1]$. We independently sample random variables $u_{i} \in U[0,1]$ for $i \in I$. These values are not known to $\mathcal{A}$ but for every match we provide the outcome to $\mathcal{A}$ based on these values. In other words, $\boldsymbol{u}=\left(u_{i}\right)_{i \in I}$ gives a sample path over random match outcomes. To describe how $\boldsymbol{u}$ translates to match outcomes, suppose that resource $i$ has been unsuccessfully matched to $s \geq 0$ arrivals in the ordered set $S=\left\{t_{1}, \cdots, t_{s}\right\}$ and $\mathcal{A}$ matches a new arrival $t_{s+1}$ to $i$. The match succeeds if $1-\prod_{j \leq s+1}\left(1-p_{i t_{j}}\right) \geq u_{i}$, and fails otherwise. Observe that $1-\prod_{j \leq s+1}\left(1-p_{i t_{j}}\right)$ is the probability that at least one of the matches to $S \cup\left\{t_{s+1}\right\}$ succeeds. Thus, $u_{i}$ is a threshold such that $i$ is successfully matched to the first arrival where the probability of success exceeds $u_{i}$.

Lemma 7. Consider a non-anticipative and deterministic algorithm $\mathcal{A}$. Let $\mathcal{A}(\omega)$ denote the matching output by $\mathcal{A}$ on sample path $\omega \in \Omega$. Similarly, let $\mathcal{A}(\boldsymbol{u})$ denote the matching output by $\mathcal{A}$ on sample path $\boldsymbol{u} \in[0,1]^{n}$ in the threshold viewpoint. For every matching $\mathcal{M}$, we have $\mathbb{P}(\mathcal{A}(\omega)=\mathcal{M})=$ $\mathbb{P}(\mathcal{A}(\boldsymbol{u})=\mathcal{M})$.

See Appendix A.1 for a proof. To connect thresholds $\boldsymbol{u}$ to the random budget viewpoint introduced in Mehta and Panigrahi (2012), we start by exploring how the matching generated by $\mathcal{A}$ changes as we vary a single threshold value while keeping all other values fixed. To this end, consider a resource $i \in I$ and fix threshold values, $u_{-i} \in[0,1]^{n-1}$, for all resources except $i$. Let $\mathcal{A}^{u_{-i}}(1)$ denote the matching output by $\mathcal{A}$ when the threshold for $i$ is 1 and threshold for every other resource is given by $u_{-i}$. Let

$$
\mathcal{A}_{i}^{u_{-i}}(1)=\left\{t_{1}, \cdots, t_{m_{\mathcal{A}}^{u}}^{u_{-i}}\right\}
$$

denote the ordered set of $m_{\mathcal{A}}^{u_{-i}}$ arrivals matched to $i$ in matching $\mathcal{A}^{u_{-i}}(1)$.

Lemma 8. Consider $i \in I, u_{-i} \in[0,1]^{n-1}$, and a deterministic non-anticipative algorithm $\mathcal{A}$. Let $\mathcal{A}_{i}^{u_{-i}}\left(u_{i}\right)$ denote the ordered set of arrivals matched to $i$ in the matching $\mathcal{A}\left(u_{i}, u_{-i}\right)$.

(i) For every $k \in\left[m_{\mathcal{A}}^{u_{-i}}\right]$, $i$ is matched to $t_{k} \in \mathcal{A}_{i}^{u_{-i}}(1)$ if and only if $u_{i}>1-\prod_{j \leq k-1}\left(1-p_{i t_{j}}\right)$. Thus, conditioned on $u_{-i}, i$ is matched to $t_{k}$ with probability $\prod_{j \leq k-1}\left(1-p_{i t_{j}}\right)$. 
(ii) For every $u_{i} \in[0,1]$, there exist some $k \in\left[m_{\mathcal{A}}^{u_{-i}}\right]$ such that $\mathcal{A}_{i}^{u_{-i}}\left(u_{i}\right)=\left\{t_{1}, \cdots, t_{k}\right\}$. Further, $\mathcal{A}_{i}^{u_{-i}}\left(u_{i}\right) \subseteq \mathcal{A}_{i}^{u_{-i}}\left(u_{i}^{\prime}\right)$ for every $0 \leq u_{i} \leq u_{i}^{\prime} \leq 1$.

A proof is provided in Appendix A.1. Next, we connect thresholds $\boldsymbol{u}$ to budgets $\left\{b_{i}\right\}_{i \in I}$.

From Thresholds to Budgets: Conditioned on thresholds $u_{-i}$, consider the set $\mathcal{A}_{i}^{u_{-i}}(1)$ of $m_{\mathcal{A}}^{u_{-i}}$ arrivals that are sequentially matched to $i$ in $\mathcal{A}$. Let

$$
p(k)=\sum_{j \leq k} p_{i t_{j}}, \forall k \in\left[m_{\mathcal{A}}^{u_{-i}}\right] .
$$

In the random budget viewpoint, for any given resource $i$, conditioned on thresholds $u_{-i}$, we independently draw a starting budget $b_{i}$ from a suitable distribution (defined later). Every time resource $i$ is matched, we reduce the remaining budget by the probability of the corresponding edge. The match is successful if the remaining budget is $\leq 0$. In other words, the first match where the sum of the probabilities of all edges matched to $i$ is greater than or equal to the starting budget $b_{i}$, is successful, and all prior matches are failures.

Conditioned on thresholds $u_{-i}$, let $F_{\mathcal{A}}^{u_{-i}}(x)$ denote the probability distribution of budget $b_{i}$. Define

$$
p_{i}=\max _{t \mid(i, t) \in E} p_{i t} \text { and } p=\max _{i \in I} p_{i} .
$$

The support of $F_{\mathcal{A}}^{u_{-i}}(x)$ must include the set $\left\{p(1), \cdots, p\left(m_{\mathcal{A}}^{u_{-i}}\right)\right\}$. In addition, we also include the set $\left\{p\left(m_{\mathcal{A}}^{u_{-i}}\right)+p_{i}, p\left(m_{\mathcal{A}}^{u_{-i}}\right)+2 p_{i}, p\left(m_{\mathcal{A}}^{u_{-i}}\right)+3 p_{i}, \cdots,+\infty\right\}$ in the support. For simplicity, let $p(\ell)=$ $p\left(m_{\mathcal{A}}^{u_{-i}}\right)+\left(\ell-m_{\mathcal{A}}^{u_{-i}}\right) p_{i}$ for every $\ell \geq m_{\mathcal{A}}^{u_{-i}}$. On $\left\{p(1), \cdots, p\left(m_{\mathcal{A}}^{u_{-i}}\right)\right\}$, the distribution is (uniquely) defined by probabilities,

$$
\mathbb{P}(x=p(k))=p_{i t_{k}} \prod_{j=1}^{k-1}\left(1-p_{i t_{j}}\right) \quad \forall k \in\left[m_{\mathcal{A}}^{u_{-i}}\right] .
$$

On $\left\{p\left(m_{\mathcal{A}}^{u_{-i}}+1\right), p\left(m_{\mathcal{A}}^{u_{-i}}+2\right), \cdots,+\infty\right\}$, we define the distribution as,

$$
\mathbb{P}\left(x=p\left(m_{\mathcal{A}}^{u_{-i}}\right)+\ell p_{i}\right)=p_{i}\left(1-P\left(x \leq p\left(m_{\mathcal{A}}^{u_{-i}}\right)\right)\right) \prod_{j=1}^{\ell-1}\left(1-p_{i}\right) \quad \forall \ell \in\{1,2, \cdots,+\infty\} .
$$

This part of the distribution can be set differently as long as, $\mathbb{P}\left(b_{i}>p\left(m_{\mathcal{A}}^{u_{-i}}\right)\right)=\prod_{j=1}^{m_{\mathcal{A}}^{u_{-i}}}\left(1-p_{i t_{j}}\right)$. We made a choice that is convenient for analysis. Let $G_{\mathcal{A}}^{u_{-i}}:[0,1] \rightarrow[0,+\infty)$ denote the inverse function such that $F_{\mathcal{A}}^{u_{-i}}\left(G_{\mathcal{A}}^{u_{-i}}(u)\right)=u$. An alternative way to generate starting budget $b_{i} \sim F_{\mathcal{A}}^{u_{-i}}$ (conditioned on $\left.u_{-i}\right)$ is as follows: Independently sample threshold $u_{i} \in[0,1]$ and set budget $b_{i}=G_{\mathcal{A}}^{u_{-i}}\left(u_{i}\right)$.

Corollary 1. Consider a non-anticipative deterministic algorithm $\mathcal{A}$, resource $i \in I$, and condition on thresholds $u_{-i} \in[0,1]^{n-1}$. Let $\mathcal{A}_{i}^{b, u_{-i}}(x)$ denote the ordered set of arrivals matched to $i$ in the budget viewpoint with starting budget $x$. Then, 
(i) $\mathcal{A}_{i}^{b, u_{-i}}\left(G_{\mathcal{A}}^{u_{-i}}(u)\right)=\mathcal{A}_{i}^{u_{-i}}(u), \forall u \in[0,1]$.

(ii) The probability that $i$ is successfully matched in $\mathcal{A}$ equals $\mathbb{P}\left(G_{\mathcal{A}}^{u_{-i}}(u) \leq p\left(m_{\mathcal{A}}^{u_{-i}}\right)\right)$.

The corollary is a direct consequence of Lemma 8 and the definition of the budget viewpoint.

Remarks: We refer to $p\left(m_{\mathcal{A}}^{u_{-i}}\right)$ as the effort threshold of $i$ conditioned on $u_{-i}$. A resource is successfully matched when its effort threshold no less than the budget. This generalizes the budget viewpoint in Mehta and Panigrahi (2012). An important difference between the generalized budget viewpoint and the special case of identical probabilities is that the distribution of threshold $b_{i}$ is a function of $u_{-i}$. For identical probabilities, the distribution is independent of $u_{-i}$. Note that while the distribution depends on $u_{-i}$, the budget is sampled independently for every $i \in I$.

\subsection{Approximation by $\operatorname{Exp}(1)$}

Now, we consider the case of small edge probabilities and show that the exponential distribution, $\operatorname{Exp}(1)$, approximates $F_{\mathcal{A}}^{u_{-i}}$. Let

$$
x=(1 \pm \delta) y \quad \Rightarrow \quad(1-\delta) y \leq x \leq(1+\delta) y .
$$

Let $O(x)$ denote $c \cdot x$, where $c$ is a constant that is independent of $x$ and the problem parameters $\left\{p_{i t}\right\}_{(i, t) \in E},\left\{r_{i}\right\}_{i \in I}, n$ and $T$. Notice that $x=(1 \pm O(\delta)) y \Leftrightarrow y=(1+O(\delta)) x$.

Lemma 9. Given a non-anticipative deterministic algorithm $\mathcal{A}$, resource $i \in I$, threshold vector $u_{-i} \in[0,1]^{n-1}$ and $p \leq 0.38$, we have,

(i) $1-F_{\mathcal{A}}^{u}(x)=\left(1 \pm O\left(\sqrt{p_{i}}\right)\right) e^{-x} \quad \forall x \in\left[0, \frac{1}{\sqrt{p_{i}}}\right]$.

(ii) $F_{\mathcal{A}}^{u_{-i}}(x)=\left(1 \pm O\left(\sqrt{p_{i}}\right)\right)\left(1-e^{-x}\right) \quad \forall x \geq 0$.

We give a self-contained proof of the lemma in Appendix A.2. Note that, it may also be possible to prove this lemma using using Le Cam's theorem (Le Cam 1960). From this lemma, we have that $\operatorname{Exp}(1)$ is a good approximation for budget distribution $F_{\mathcal{A}}^{u_{-i}}$ when the effort threshold $p\left(m_{\mathcal{A}}^{u_{-i}}\right) \leq$ $1 / \sqrt{p_{i}}$. This approximation will play an important role in analyzing Algorithm 2. The next lemma gives another useful means of approximating the true distribution of budgets by $\operatorname{Exp}(1)$. In the lemma, we use $E_{u_{i}}[\cdot]$ to denote expectation w.r.t. the randomness in threshold $u_{i} \sim U[0,1]$. A proof of the lemma is provided in Appendix A.2.

Lemma 10. Consider a deterministic non-anticipative algorithm $\mathcal{A}$, resouce $i \in I$, threshold vector $u_{-i} \in[0,1]^{n-1}$, a non-decreasing integrable function $h: \mathbb{R}^{+} \rightarrow \mathbb{R}^{+}$such that,

(i) $h(0)=0$,

(ii) $h(x)=h\left(\frac{1}{\sqrt{p_{i}}}\right) \forall x \geq \frac{1}{\sqrt{p_{i}}}$. 
For $p \leq 0.38$, we have,

$$
E_{u_{i}}\left[h\left(G_{\mathcal{A}}^{u_{-i}}\left(u_{i}\right)\right)\right]=\left(1 \pm O\left(\sqrt{p_{i}}\right)\right) E_{b \sim \operatorname{Exp}(1)}[h(b)] .
$$

Recall that both Algorithm 2 and the offline benchmark are deterministic. Therefore, the lemmas in this section and in Section 4.1, apply to both Algorithm 2 and the offline benchmark. In the next section, we present a new path-based linear system and show that proving feasibility of the system establishes a competitive ratio guarantee for Algorithm 2. In Section 4.4, we use the alternative viewpoint of stochastic rewards (and its approximation) to prove feasibility of this system.

\subsection{LP Free Framework}

In this section, we define a system of linear inequalities such that the existence of a feasible solution to this system implies a competitive ratio guarantee for Algorithm 2. Our linear system has a single constraint for every offline vertex as opposed to a constraint for every edge. The new feasibility constraints are path-based and inspired by duality but do not necessarily correspond to the dual of a program. This makes the analysis LP free and generally speaking, robust to any issues related to tightness of a mathematical program. We start by introducing notation.

Let OPT denote the fully offline algorithm as well as its expected total reward. Similarly, let ALG denote denote Algorithm 2 and its expected reward. Given a resource $i \in I$, and thresholds $u_{-i} \in[0,1]^{n-1}$, let $p\left(m_{\mathrm{ALG}}^{u_{-i}}\right)$ and $p\left(m_{\mathrm{OPT}}^{u_{-i}}\right)$ denote the effort threshold of resource $i$ in ALG and OPT respectively. We use $E_{\boldsymbol{u}}[\cdot]$ to denote expectation w.r.t. the randomness in thresholds $\boldsymbol{u}$. For every $i \in I$, let $E_{u_{-i}}[\cdot]$ denote expectation w.r.t. randomness in $u_{-i}$.

The variables of the system are $\lambda_{i t}^{u_{-i}}$ and $\theta_{i}^{u_{-i}}$, for every $u_{-i} \in[0,1]^{n-1}, i \in I,(i, t) \in E$. The system is defined as follows,

$$
\begin{array}{r}
\theta_{i}^{u_{-i}}+\sum_{t \mid(i, t) \in E} \lambda_{i t}^{u_{-i}} \geq \alpha r_{i}\left(1-e^{-p\left(m_{\mathrm{OPP}}^{u_{-i}}\right)}\right) \quad \forall i \in I, u_{-i} \in[0,1]^{n-1}, \\
\beta \mathrm{ALG} \geq \sum_{i \in I} E_{u_{-i}}\left[\theta_{i}^{u_{-i}}\right]+\sum_{(i, t) \in E} E_{u_{-i}}\left[\lambda_{i t}^{u_{-i}}\right], \\
\theta_{i}^{u_{-i}}, \lambda_{i t}^{u_{-i}} \geq 0 \quad \forall u_{-i} \in[0,1]^{n-1}, i \in I, t \in T,
\end{array}
$$

where $\alpha, \beta \geq 0$ are constants that determine the competitive ratio guarantee. Linearity of the system follows from linearity of the expectation operator. In Appendix B, we compare our LP free framework with the LP based primal-dual approach employed in Huang and Zhang (2020).

The next lemma shows that the feasibility of this linear system implies a competitive ratio guarantee for ALG.

Lemma 11. Given a feasible solution to the system defined by (13), (14), and (15), we have, $\mathrm{ALG} \geq \frac{\alpha}{\beta} \mathrm{OPT}$. 
Proof. Given $i \in I$ and $u_{-i} \in[0,1]^{n-1}$, let $\mathrm{OPT}_{i}$ denote the expected reward of OPT from successfully matching $i$. Using Corollary 1 (ii) and Lemma 9(ii), we have,

$$
\mathrm{OPT}_{i}=\left(1 \pm O\left(\sqrt{p_{i}}\right)\right) r_{i} E_{u_{-i}}\left[1-e^{-p\left(m_{\mathrm{OPT}}^{u_{-i}}\right)}\right] .
$$

Thus, given a feasible solution to the system, we have,

$$
\left(1-O\left(\sqrt{p_{i}}\right)\right) \alpha \mathrm{OPT}_{i} \leq E_{u_{-i}}\left[\theta_{i}^{u_{-i}}+\sum_{t \mid(i, t) \in E} \lambda_{i t}^{u_{-i}}\right] \quad \forall i \in I
$$

Summing up these inequalities over $i \in I$, we get,

$$
\begin{aligned}
(1-O(\sqrt{p})) \alpha \mathrm{OPT} & \leq \sum_{i \in I} E_{u_{-i}}\left[\theta_{i}^{u_{-i}}+\sum_{t \mid(i, t) \in E} \lambda_{i t}^{u_{-i}}\right] \\
& \leq \sum_{i \in I} E_{u_{-i}}\left[\theta_{i}^{u_{-i}}\right]+\sum_{(i, t) \in E} E_{u_{-i}}\left[\lambda_{i t}^{u_{-i}}\right] \\
& \leq \beta \mathrm{ALG} .
\end{aligned}
$$

\subsection{Finding a Feasible Solution}

In this section, we perform a dual fitting i.e., using the matching generated by ALG and OPT, we construct a feasible solution to the system given by (13), (14), and (15). To define the path-based candidate solution, we couple the randomness in ALG and OPT by choosing the same thresholds $\boldsymbol{u}$ for both algorithms. We start by introducing new terminology and notation that we use to define the candidate solution.

Truncating OPT: Consider a non-anticipative deterministic algorithm OPT ${ }^{\text {tr }}$ that executes OPT but leaves some arrivals unmatched, resulting in an effort threshold of at most $\frac{1}{\sqrt{p}} \forall i \in I$. For $i \in I$ and $t \in T$, let $l_{i}(\mathrm{OPT}, t)$ denote the sum of probabilities of all edges matched to $i$ (in OPT) prior to the match to $t$. Then, for every match $(i, t)$ made by OPT, the truncated algorithm $\mathrm{OPT}^{t r}$ matches $t$ as follows,

1. If $l_{i}(\mathrm{OPT}, t) \leq 1 / \sqrt{p}$, then match $t$ to $i$.

2. If $l_{i}(\mathrm{OPT}, t)>1 / \sqrt{p}$, then leave $t$ unmatched.

Observe that for every resource $i$ and (partial) sample path $u_{-i}$, the effort threshold of $i$ in OPT ${ }^{t r}$ i.e., $p\left(m_{\mathrm{OPT}^{t r}}^{u_{-i}}\right)$, is at most $\frac{1}{\sqrt{p}}$. The next lemma states that truncation has a negligible effect on the expected total revenue. See Appendix A.3 for a proof.

Lemma 12. For every $i \in I$, let $\mathrm{OPT}_{i}$ and $\mathrm{OPT}_{i}^{t r}$ denote the expected total revenue from matching resource $i$ in $\mathrm{OPT}$ and in $\mathrm{OPT}^{\text {tr }}$ respectively. Then, we have, $\mathrm{OPT}_{i}^{t r} \geq(1-O(\sqrt{p})) \mathrm{OPT}_{i} \forall i \in I$. 
Let $\mathbb{1}(A)$ be an indicator for event $A$. Define,

$$
\operatorname{tr}(x)=\min \left\{x, \frac{1}{\sqrt{p}}\right\} \quad \forall x \in \mathbb{R}
$$

Let $\mathrm{OPT}_{i}^{t r}\left(u_{-i}, b\right)$ denote the set of arrivals matched to $i$ in $\mathrm{OPT}^{t r}$ on sample path given by thresholds $u_{-i}$ and budget $b$ for resource $i$. Recall that we use a function $g(\cdot)$ in Algorithm 2 to compute the reduced rewards for resources. We define the candidate solution through functions $z_{i t}^{u_{-i}}: \mathbb{R}^{+} \times \mathbb{R}^{+} \rightarrow[0,1]$ and $v_{i}^{u_{-i}}: \mathbb{R}^{+} \rightarrow[0,1]$. For every $i \in I,(i, t) \in E, u_{-i} \in[0,1]^{n-1}$, let

$$
\begin{aligned}
& z_{i t}^{u_{-i}}(x, y)=p_{i t} r_{i} g\left(\operatorname{tr}\left(p\left(m_{\mathrm{ALG}}^{u_{-i}}\right)\right)\right) \times \mathbb{1}\left(t \in \mathrm{OPT}_{i}^{t r}\left(u_{-i}, x\right)\right) \times \mathbb{1}\left(y>\operatorname{tr}\left(p\left(m_{\mathrm{ALG}}^{u_{-i}}\right)\right)\right) \\
& v_{i}^{u_{-i}}(x)=r_{i} \int_{0}^{\min \left\{x, \operatorname{tr}\left(p\left(m_{\mathrm{ALG}}^{u_{-i}}\right)\right)\right\}}(1-g(y)) d y .
\end{aligned}
$$

At a high level, these functions split up the total reward of ALG. The function $z_{i t}^{u_{-i}}(x, y)$ is a lower bound on the reduced reward of resource $i$ seen by arrivals matched to $i$ in OPT. When the budget of $i$ in ALG is "large", $i$ is not successfully matched and is available at every arrival. In such a scenario, $z_{i t}^{u_{-i}}(x, y)$ is non-zero at all arrivals $t \in \mathrm{OPT}_{i}^{t r}\left(u_{-i}, x\right)$. Now, our candidate solution is defined as,

$$
\lambda_{i t}^{u_{-i}}=E_{b \sim \operatorname{Exp}(1)}\left[z_{i t}^{u_{-i}}(b, b)\right] \quad \text { and } \quad \theta_{i}^{u_{-i}}=E_{b \sim \operatorname{Exp}(1)}\left[v_{i}^{u_{-i}}(b)\right] .
$$

The solution is non-negative by definition, satisfying (15). To show the validity of this candidate solution, we first show that inequality (14) is satisfied for a sufficiently small value of $\beta$ (Lemma 13). Then, we show that inequalities (13) are satisfied for a large enough value of $\alpha$ (Lemma 14). We establish these claims for the following family of functions $g(\cdot)$.

Valid functions: Function $g:[0, \infty) \rightarrow[0,1]$ is valid if it satisfies the following properties,

(a) $g(\cdot)$ is a strictly decreasing and differentiable function.

(b) There exists a constant $\eta \geq 0$, such that, $g(x) \leq(1+\eta \sqrt{\epsilon}) g(x+\epsilon) \quad \forall \epsilon \leq 1, x \geq 0$.

(c) There exists a constant $c \geq 0$, such that, $g(x) \leq \frac{c}{x} \quad \forall x>0$.

(d) $\min _{x \geq 0} f(x)=g(0)$, where,

$$
f(x)=1-e^{-x}[1-g(x)(x+1)]-\int_{0}^{x} g(z) e^{-z} d z .
$$

Lemma 13. For any valid function $g$ and $p \leq 0.38$, the candidate solution given by (18) satisfies constraint (14) with $\beta=1+O(\sqrt{p})$.

Proof. We first present an alternative way to write the expected total reward of ALG. Let ALG $(\boldsymbol{u})$ denote the matching generated by ALG given thresholds $\boldsymbol{u}$. Let $l_{i}^{\boldsymbol{u}}(t)$ denote the sum of 
probabilities of edges in $\operatorname{ALG}(\boldsymbol{u})$ that are incident on $i$ prior to $t$. For every edge $(i, t) \in \operatorname{ALG}(\boldsymbol{u})$, let

$$
\begin{aligned}
& f_{t}^{\boldsymbol{u}}=r_{i} \int_{l_{i}^{\boldsymbol{u}}(t)}^{l_{i}^{\boldsymbol{u}}(t)+p_{i t}} g(y) d y, \\
& h_{i t}^{\boldsymbol{u}}=r_{i} \int_{l_{i}^{\boldsymbol{u}}(t)}^{l_{i}^{\boldsymbol{u}}(t)+p_{i t}}(1-g(y)) d y .
\end{aligned}
$$

Then, for every $(i, t) \in \operatorname{ALG}(\boldsymbol{u})$ we have, $f_{t}^{\boldsymbol{u}}+h_{i t}^{\boldsymbol{u}}=p_{i t} r_{i}$. We claim that,

$$
\mathrm{ALG}=E_{\boldsymbol{u}}\left[\sum_{t \in T} f_{t}^{\boldsymbol{u}}+\sum_{(i, t) \in \mathrm{ALG}(\boldsymbol{u})} h_{i t}^{\boldsymbol{u}}\right] .
$$

The proof of this claim is a generalization of Lemma 2 in Mehta and Panigrahi (2012). Consider a variant of the stochastic rewards setting where resource usage is stochastic but rewards are deterministic. Given a match $(i, t)$ in the variant, resource $i$ is consumed (unavailable for future matches) with probability $p_{i t}$ but we earn a reward $p_{i t} r_{i}$ with probability 1 . By coupling the thresholds $\boldsymbol{u}$ in this variant and the original setting, and using the linearity of expectation, we have that the overall reward of a non-anticipative algorithm $\mathcal{A}$ (such as ALG), is the same in both settings. Observe that, $E_{\boldsymbol{u}}\left[\sum_{t \in T} f_{t}^{\boldsymbol{u}}+\sum_{(i, t) \in \mathrm{ALG}(\boldsymbol{u})} h_{i t}^{\boldsymbol{u}}\right]$ is the expected reward of ALG on the variant problem, giving us the desired. Now, to prove the main claim it suffices to show that,

$$
\begin{aligned}
& \sum_{i \in I} E_{u_{-i}}\left[\theta_{i}^{u_{-i}}\right] \leq(1+O(\sqrt{p})) \sum_{(i, t) \in \operatorname{ALG}(\boldsymbol{u})} E_{\boldsymbol{u}}\left[h_{i t}^{\boldsymbol{u}}\right] \\
& \sum_{(i, t) \in E} E_{u_{-i}}\left[\lambda_{i t}^{u_{-i}}\right] \leq(1+O(\sqrt{p})) E_{\boldsymbol{u}}\left[\sum_{t \in T} f_{t}^{\boldsymbol{u}}\right]+O(\sqrt{p}) \mathrm{ALG} .
\end{aligned}
$$

Proof of (19): For every $i \in I$ and $u_{-i} \in[0,1]^{n-1}$, observe that $v_{i}^{u_{-i}}(x)$ meets the criteria in Lemma 10 - it is integrable and non-decreasing in $x$, has a constant value for $x \geq \frac{1}{\sqrt{p_{i}}}$, and $v_{i}^{u_{-i}}(0)=0$. Applying the lemma we get,

$$
\theta_{i}^{u_{-i}}=\left(1 \pm O\left(\sqrt{p_{i}}\right)\right) E_{u_{i}}\left[v_{i}^{u_{-i}}\left(G_{\mathrm{ALG}}^{u_{-i}}\left(u_{i}\right)\right)\right] \quad i \in I, u_{-i} \in[0,1]^{n-1} .
$$

Now, to prove (19), it suffices to show that $\sum_{i \in I} v_{i}^{u_{-i}}\left(G_{\mathrm{ALG}}^{u_{-i}}\left(u_{i}\right)\right) \leq \sum_{i \in I} \sum_{t \in \mathrm{ALG}_{i}(\boldsymbol{u})} h_{i t}^{\boldsymbol{u}} \quad \forall \boldsymbol{u} \in[0,1]^{n}$. In fact, we show the following stronger statement. Consider an arbitrary $i \in I$ and $\boldsymbol{u} \in[0,1]^{n}$. Let $\operatorname{ALG}_{i}(\boldsymbol{u})$ denote the set of arrivals matched to $i$ in matching $\operatorname{ALG}(\boldsymbol{u})$. Then,

$$
\begin{aligned}
\sum_{t \in \mathrm{ALG}_{i}(\boldsymbol{u})} h_{i t}^{u} & =r_{i} \sum_{t \in \mathrm{ALG}_{i}(\boldsymbol{u})} \int_{l_{i}^{u}(t)}^{l_{i}^{u}(t)+p_{i t}}(1-g(y)) d y \\
& =r_{i} \int_{0}^{\min \left\{G_{\mathrm{ALG}}^{u_{-i}}\left(u_{i}\right), p\left(m_{\mathrm{ALG}}^{u_{-i}}\right)\right\}}(1-g(y)) d y \\
& \geq v_{i}^{u_{-i}}\left(G_{\mathrm{ALG}}^{u_{-i}}\left(u_{i}\right)\right) .
\end{aligned}
$$


The first equality and the inequality follow by definition. Second equality follows from the fact that $\max _{t \in \mathrm{ALG}_{i}(\boldsymbol{u})} l_{i}^{\boldsymbol{u}}(t)+p_{i t}$, which is the sum of probabilities of all edges incident on $i$ in $\operatorname{ALG}(\boldsymbol{u})$, is equal to $\min \left\{G_{\mathrm{ALG}}^{u_{-i}}\left(u_{i}\right), p\left(m_{\mathrm{ALG}}^{u_{-i}}\right)\right\}$. This completes the proof of (19).

Proof of (20): The proof consists of two parts. First, we show that,

$$
\lambda_{i t}^{u_{-i}} \leq\left(1+O\left(\sqrt{p_{i}}\right)\right) E_{u_{i}}\left[z_{i t}^{u_{-i}}\left(G_{\mathrm{OPT}}^{u_{-i}}\left(u_{i}\right), G_{\mathrm{ALG}}^{u_{-i}}\left(u_{i}\right)\right)\right] \quad \forall(i, t) \in E, u_{-i} \in[0,1]^{n-1} .
$$

Then, we show,

$$
\sum_{(i, t) \in E} E_{\boldsymbol{u}}\left[z_{i t}^{u_{-i}}\left(G_{\mathrm{OPT}}^{u_{-i}}\left(u_{i}\right), G_{\mathrm{ALG}}^{u_{-i}}\left(u_{i}\right)\right)\right] \leq(1+O(\sqrt{p})) E_{\boldsymbol{u}}\left[\sum_{t \in T} f_{t}^{u}\right]+O(\sqrt{p}) \mathrm{ALG} .
$$

Combining these inequalities gives us the desired.

Given an edge $(i, t)$ and (partial) sample path $u_{-i}$, observe that,

$$
z_{i t}^{u_{-i}}\left(G_{\mathrm{OPT}}^{u_{-i}}\left(u_{i}\right), G_{\mathrm{ALG}}^{u_{-i}}\left(u_{i}\right)\right)=c \mathbb{1}\left(A\left(u_{i}\right)\right) \mathbb{1}\left(B\left(u_{i}\right)\right),
$$

here $c$ is a constant independent of $u_{i}, A\left(u_{i}\right)$ corresponds to the event that $t \in \operatorname{OPT}_{i}^{t r}\left(u_{-i}, G_{\mathrm{OPT}}^{u_{-i}}\left(u_{i}\right)\right)$, and $B\left(u_{i}\right)$ is the event, $G_{\mathrm{ALG}}^{u_{-i}}\left(u_{i}\right)>\operatorname{tr}\left(p\left(m_{\mathrm{ALG}}^{u_{-i}}\right)\right)$. From Lemma $8(i i)$ and Corollary $1(i)$, we have that for some $u_{A}, u_{B} \in[0,1]$ (independent of $u_{i}$ ), event $A\left(u_{i}\right)$ and event $B\left(u_{i}\right)$ are equivalent to events $u_{i} \geq u_{A}$ and $u_{i} \geq u_{B}$ respectively. For brevity, let

$$
Z_{i t}^{u_{-i}}\left(u_{i}\right)=z_{i t}^{u_{-i}}\left(G_{\mathrm{OPT}}^{u_{-i}}\left(u_{i}\right), G_{\mathrm{ALG}}^{u_{-i}}\left(u_{i}\right)\right)=c \mathbb{1}\left(u_{i}>u_{A}\right) \mathbb{1}\left(u_{i}>u_{B}\right) .
$$

Define random variables $X_{A}\left(u_{i}\right)=c \mathbb{1}\left(u_{i}>u_{A}\right)$ and $X_{B}\left(u_{i}\right)=c \mathbb{1}\left(u_{i}>u_{B}\right)$. Then,

$$
\begin{aligned}
E_{u_{i}}\left[z_{i t}^{u_{-i}}\left(u_{i}\right)\right]= & \mathbb{1}\left(u_{A} \geq u_{B}\right) E_{u_{i}}[ \\
= & \left(1 \pm O\left(\sqrt{p_{i}}\right)\right) c\left[\mathbb{1}\left(u_{A} \geq u_{B}\right) E_{b \sim \operatorname{Exp}(1)}\left[\mathbb{1}\left(b>G_{\mathrm{OPT}}^{u_{-i}}\left(u_{A}\right)\right)\right]\right. \\
& \quad+\mathbb{1}\left(u_{A}<u_{B}\right) E_{b \sim \operatorname{Exp}(1)}\left[\mathbb{1}\left(b>u_{\mathrm{ALG}}\right) E_{u_{i}}\left[X_{B}\left(u_{i}\right)\right]\right. \\
& \\
\geq & \left(1-O\left(\sqrt{p_{i}}\right)\right) E_{b \sim \operatorname{Exp}(1)}\left[z_{i t}^{u_{-i}}(b, b)\right] \\
= & \left(1-O\left(\sqrt{p_{i}}\right)\right) \lambda_{i t}^{u_{-i}} .
\end{aligned}
$$

The second equality follows from Lemma 10. The inequality follows from the fact that for every $b \geq 0$, both $\mathbb{1}\left(b>G_{\mathrm{OPT}}^{u_{-i}}\left(u_{A}\right)\right)$ and $\mathbb{1}\left(b>G_{\mathrm{ALG}}^{u_{-i}}\left(u_{B}\right)\right)$ are lower bounded by $\mathbb{1}\left(b>G_{\mathrm{OPT}}^{u_{-i}}\left(u_{A}\right)\right) \mathbb{1}(b>$ $\left.G_{\mathrm{ALG}}^{u_{-i}}\left(u_{B}\right)\right)$.

Now, we prove the desired upper bound on $\sum_{(i, t) \in E} E_{\boldsymbol{u}}\left[Z_{i t}^{u_{-i}}\left(u_{i}\right)\right]$. Given sample path $\boldsymbol{u}$, we partition resources $i \in I$ into two categories - normal and extreme. A resource $i$ is normal if the effort threshold $p\left(m_{\mathrm{ALG}}^{u_{-i}}\right) \leq 1 / \sqrt{p}$. Let $I_{1}(\boldsymbol{u})$ denote the set of normal resources. Let $I_{2}(\boldsymbol{u})=I \backslash I_{1}(\boldsymbol{u})$, denote the set of extreme resources. 
Consider an arbitrary sample path $\boldsymbol{u}$ and an arrival $t \in T$. Let $i^{*}$ denote the normal resource (if any) matched to $t$ in the matching $\operatorname{OPT}^{t r}(\boldsymbol{u})$. Then,

$$
\sum_{i \mid i \in I_{1}(\boldsymbol{u}),(i, t) \in E} Z_{i t}^{u_{-i}}\left(u_{i}\right)=r_{i^{*}} p_{i^{*} t} g\left(p\left(m_{\mathrm{ALG}}^{u^{-i^{*}}}\right)\right) \mathbb{1}\left(G_{\mathrm{ALG}}^{u^{-i^{*}}}\left(u_{i}\right)>p\left(m_{\mathrm{ALG}}^{u^{-i^{*}}}\right)\right) \leq(1+O(\sqrt{p})) f_{t}^{\boldsymbol{u}},
$$

here the last inequality follows from the fact that ALG is greedy w.r.t. expected reduced rewards and property (b) of valid functions. Thus,

$$
\sum_{(i, t) \in E, i \in I_{1}(\boldsymbol{u})} Z_{i t}^{u_{-i}}\left(u_{i}\right)=\sum_{t \in T} \sum_{i \mid i \in I_{1}(\boldsymbol{u}),(i, t) \in E} Z_{i t}^{u_{-i}}\left(u_{i}\right) \leq(1+O(\sqrt{p})) \sum_{t \in T} f_{t}^{\boldsymbol{u}} \forall \boldsymbol{u} \in[0,1]^{n} .
$$

For extreme resources, we have the following upper bound for every $u_{-i} \in[0,1]^{n-1}$,

$$
\begin{aligned}
\sum_{(i, t) \in E, i \in I_{2}(\boldsymbol{u})} Z_{i t}^{u_{-i}}\left(u_{i}\right) & =\sum_{i \in I_{2}(\boldsymbol{u})}\left[r_{i} g\left(\frac{1}{\sqrt{p}}\right) \times \mathbb{1}\left(G_{\mathrm{ALG}}^{u_{-i}}\left(u_{i}\right)>\frac{1}{\sqrt{p}}\right) \times \sum_{t \in \mathrm{OPT}_{i}^{t r}\left(u_{-i}, G_{\mathrm{OPP}}^{u_{-i}}\left(u_{i}\right)\right)} p_{i t}\right] \\
& \stackrel{(A)}{\leq} \sum_{i \in I_{2}(\boldsymbol{u})} r_{i} \frac{g\left(\frac{1}{\sqrt{p}}\right)}{\sqrt{p}} \mathbb{1}\left(G_{\mathrm{ALG}}^{u_{-i}}\left(u_{i}\right)>\frac{1}{\sqrt{p}}\right) \\
& \stackrel{(B)}{\leq} O(1) \sum_{i \in I_{2}(\boldsymbol{u})} r_{i} \mathbb{1}\left(G_{\mathrm{ALG}}^{u_{-i}}\left(u_{i}\right)>\frac{1}{\sqrt{p}}\right) .
\end{aligned}
$$

Inequality $(A)$ follows from the fact that $\sum_{t \in \mathrm{OPT}_{i}^{t r}\left(u_{-i}, G_{\mathrm{OPT}}^{\left.u_{-i}\left(u_{i}\right)\right)}\right.} p_{i t} \leq \operatorname{tr}\left(G_{\mathrm{OPT}}^{u_{-i}}\left(u_{i}\right)\right) \leq \frac{1}{\sqrt{p}}$. Inequality $(B)$ follows from property $(c)$ of valid functions. Combining the upper bounds for normal and extreme resources, we get,

$$
\begin{aligned}
E_{\boldsymbol{u}}\left[\sum_{(i, t) \in E} Z_{i t}^{u_{-i}}\left(u_{i}\right)\right]= & E_{\boldsymbol{u}}\left[\sum_{(i, t) \in E, i \in I_{1}(\boldsymbol{u})} Z_{i t}^{u_{-i}}\left(u_{i}\right)\right]+E_{\boldsymbol{u}}\left[\sum_{(i, t) \in E, i \in I_{2}(\boldsymbol{u})} Z_{i t}^{u_{-i}}\left(u_{i}\right)\right] \\
\leq & (1+O(\sqrt{p})) E_{\boldsymbol{u}}\left[\sum_{t} f_{t}^{\boldsymbol{u}}\right] \\
& +O(1) \sum_{i \in I} r_{i} E_{\boldsymbol{u}}\left[\mathbb{1}\left(i \in I_{2}(\boldsymbol{u})\right) \times \mathbb{1}\left(G_{\mathrm{ALG}}^{u_{-i}}\left(u_{i}\right)>\frac{1}{\sqrt{p}}\right)\right]
\end{aligned}
$$

It remains to show that $\sum_{i \in I} r_{i} E_{\boldsymbol{u}}\left[\mathbb{1}\left(i \in I_{2}(\boldsymbol{u})\right) \times \mathbb{1}\left(G_{\mathrm{ALG}}^{u_{-i}}\left(u_{i}\right)>\frac{1}{\sqrt{p}}\right)\right] \leq O(\sqrt{p}) \mathrm{ALG}$.

$$
\begin{aligned}
& \sum_{i \in I} r_{i} E_{\boldsymbol{u}}\left[\mathbb{1}\left(i \in I_{2}(\boldsymbol{u})\right) \times \mathbb{1}\left(G_{\mathrm{ALG}}^{u_{-i}}\left(u_{i}\right)>\frac{1}{\sqrt{p}}\right)\right] \\
& =\sum_{i \in I} r_{i} E_{u_{-i}}\left[\mathbb{1}\left(p\left(m_{\mathrm{ALG}}^{u_{-i}}\right)>\frac{1}{\sqrt{p}}\right) \times E_{u_{i}}\left[\mathbb{1}\left(G_{\mathrm{ALG}}^{u_{-i}}\left(u_{i}\right)>\frac{1}{\sqrt{p}}\right)\right]\right] \\
& \leq \sum_{i \in I} r_{i} E_{u_{-i}}\left[\mathbb{1}\left(p\left(m_{\mathrm{ALG}}^{u_{-i}}\right)>\frac{1}{\sqrt{p}}\right)\right] \times O(\sqrt{p}) \\
& \leq O(\sqrt{p}) \sum_{i \in I} r_{i} E_{u_{-i}}\left[\mathbb{1}\left(p\left(m_{\mathrm{ALG}}^{u_{-i}}\right)>\frac{1}{\sqrt{p}}\right) \times \frac{E_{u_{i}}\left[\mathbb{1}\left(G_{\mathrm{ALG}}^{u_{-i}}\left(u_{i}\right) \leq \frac{1}{\sqrt{p}}\right)\right]}{1-O(\sqrt{p})}\right]
\end{aligned}
$$




$$
\begin{aligned}
& \leq O(\sqrt{p}) \sum_{i \in I} r_{i} E_{u_{-i}}\left[E_{u_{i}}\left[\mathbb{1}\left(G_{\mathrm{ALG}}^{u_{-i}}\left(u_{i}\right)<p\left(m_{\mathrm{ALG}}^{u_{-i}}\right)\right)\right]\right] \\
& =O(\sqrt{p}) \mathrm{ALG}
\end{aligned}
$$

here we used the fact that $F_{\mathrm{ALG}}^{u_{-i}}(x)=(1 \pm O(\sqrt{p}))\left(1-e^{-x}\right)$ from Lemma $9(i i)$, and the approximation, $1-e^{-\frac{1}{\sqrt{p}}} \geq(1-O(\sqrt{p}))$.

Lemma 14. Given a valid function $g$, inequalities (13) are satisfied with $\alpha=(1-O(\sqrt{p})) g(0)$.

Proof. Fix an arbitrary resource $i$ sample path $u_{-i}$. To establish (13), we show that,

$$
\theta_{i}^{u_{-i}}+\sum_{t \mid(i, t) \in E} \lambda_{i t}^{u_{-i}} \geq g(0)\left(1-e^{-p\left(m_{\mathrm{OPT} t r}^{u_{-i}}\right)}\right) .
$$

Then, using Lemma 12 completes the proof.

To prove (21), we first find lower bounds on $\theta_{i}^{u_{-i}}$ and $\sum_{t \mid(i, t) \in E} \lambda_{i t}^{u_{-i}}$ separately. For brevity, we omit $u_{-i}$ from notation. In particular, let $\theta_{i}$ and $\lambda_{i t}$ denote the candidate solution $\theta_{i}^{u_{-i}}, \lambda_{i t}^{u_{-i}}$. Let $\mathrm{OPT}_{i}^{t r}(b)$ denote the set $\mathrm{OPT}_{i}^{t r}\left(u_{-i}, b\right)$ of arrivals matched to $i$ in $\mathrm{OPT}^{t r}$ on sample path given by $\left(u_{-i}, b\right)$. Let $\tau_{O}$ denote the effort threshold $p\left(m_{\mathrm{OPT}^{t r}}^{u_{-i}}\right)=\operatorname{tr}\left(p\left(m_{\mathrm{OPT}^{t r}}^{u_{-i}}\right)\right)$. Similarly, let $\tau_{A}$ denote the effort threshold $\operatorname{tr}\left(p\left(m_{\mathrm{ALG}}^{u_{-i}}\right)\right)$. Let $G(z)=\int_{0}^{z} g(x) d x$. Then, we have,

$$
\begin{aligned}
& \theta_{i}=r_{i} E_{b \sim \operatorname{Exp}(1)}\left[\int_{0}^{\min \left\{b, \tau_{A}\right\}}(1-g(y)) d y\right] \\
& =r_{i}\left[\int_{0}^{\tau_{A}}\left(z-\int_{0}^{z} g(y) d y\right) e^{-z} d z+\int_{\tau_{A}}^{+\infty}\left(\tau_{A}-\int_{0}^{\tau_{A}} g(y) d y\right) e^{-z} d z\right] \\
& =r_{i}\left[-\tau_{A} e^{-\tau_{A}}+1-e^{-\tau_{A}}-\int_{0}^{\tau_{A}}(G(z)-G(0)) e^{-z} d z\right. \\
& \left.+\tau_{A} e^{-\tau_{A}}-\left(G\left(\tau_{A}\right)-G(0)\right) e^{-\tau_{A}}\right] \\
& =r_{i}\left[1-e^{-\tau_{A}}-\int_{0}^{\tau_{A}} g(z) e^{-z} d z\right] \text {. } \\
& \sum_{t \mid(i, t) \in E} \lambda_{i t}=r_{i} g\left(\tau_{A}\right) \sum_{t \mid(i, t) \in E} p_{i t} E_{b \sim \operatorname{Exp}(1)}\left[\mathbb{1}\left(t \in \mathrm{OPT}_{i}^{t r}(b)\right) \mathbb{1}\left(b>\tau_{A}\right)\right] \\
& =r_{i} g\left(\tau_{A}\right) \int_{\tau_{A}}^{\infty}\left(\sum_{t \in \mathrm{OPT}_{i}^{t r}(b)} p_{i t}\right) e^{-z} d z \\
& \geq r_{i} g\left(\tau_{A}\right) \int_{\tau_{A}}^{\infty} \min \left\{\tau_{O}, z\right\} e^{-z} d z \\
& =r_{i} g\left(\tau_{A}\right)\left[\int_{\tau_{A}}^{\max \left\{\tau_{O}, \tau_{A}\right\}} z e^{-z} d z+\tau \int_{\max \left\{\tau_{O}, \tau_{A}\right\}}^{\infty} e^{-z} d z\right] \\
& =r_{i} g\left(\tau_{A}\right) \times \begin{cases}\tau_{O} e^{-\tau_{A}} & \tau_{O} \leq \tau_{A}, \\
\tau_{A} e^{-\tau_{A}}+e^{-\tau_{A}}-e^{-\tau_{O}} & \tau_{O}>\tau_{A} .\end{cases}
\end{aligned}
$$


Combining (22) and (23), consider the function,

$$
H\left(\tau_{O}, \tau_{A}\right):=1-e^{-\tau_{A}}-\int_{0}^{\tau_{A}} g(z) e^{-z} d z+g\left(\tau_{A}\right) \times \begin{cases}\tau_{O} e^{-\tau_{A}} & \tau_{O} \leq \tau_{A}, \\ \tau_{A} e^{-\tau_{A}}+e^{-\tau_{A}}-e^{-\tau_{O}} & \tau_{O}>\tau_{A},\end{cases}
$$

and its derivative,

$$
\begin{aligned}
\frac{\partial H\left(\tau_{O}, \tau_{A}\right)}{\partial \tau_{O}} & \leq g\left(\tau_{A}\right) \times \begin{cases}e^{-\tau_{A}} & \tau_{O} \leq \tau_{A} \\
e^{-\tau_{O}} & \tau_{O}>\tau_{A}\end{cases} \\
& <g(0) e^{-\tau_{O}} \quad \forall \tau_{A}>0
\end{aligned}
$$

here the last inequality uses the fact that $g$ is strictly decreasing (property (a) of valid functions). To prove (21), it suffices to lower bound the function,

$$
h(x, y)=\frac{H(x, y)}{\left(1-e^{-x}\right)} \quad \forall x, y \geq 0
$$

Using property (d) of valid functions, we have that $\min _{y \geq 0} h(\infty, y)=\min _{x \geq 0} f(x)=g(0)$. Also, $h(x, 0)=g(0)$ for all $x \geq 0$. Thus, if there exists a pair of values $x, y$ such that $h(x, y)<g(0)$, then $x$ must be finite and $y$ must be strictly larger than 0 . Consider one such pair $x_{1}, y_{1}$. Since $\frac{\partial H\left(x, y_{1}\right)}{\partial x}<g(0) e^{-x}=g(0) \frac{d\left(1-e^{-x}\right)}{d x}$, we have that,

$$
h\left(\infty, y_{1}\right)<h\left(x_{1}, y_{1}\right)<g(0)
$$

This contradicts the fact that $\min _{y \geq 0} h(\infty, y)=g(0)$. Thus, $h(x, y) \geq g(0)$ for all values $x, y \geq 0$.

Proof of Theorem 2. Given a valid function $g$ and $p \leq 0.38$, from Lemma 13 and Lemma 14 we have that the candidate solution given by (18) is a feasible solution to the LP free system with $\alpha=(1-O(\sqrt{p})) g(0)$ and $\beta=1+O(\sqrt{p})$. Then, using Lemma 11, we have that ALG is $(1-O(\sqrt{p})) g(0)$ competitive.

It remains to find a valid function such that $g(0) \geq 0.596$. Consider the function $g(x)=$ $e^{x+1} \int_{x+1}^{\infty} \frac{e^{-y}}{y} d y$. This is a strictly decreasing and differentiable function (property (a) of valid functions) with $0.5963 \leq g(0) \leq 0.5964$ (bounds numerically evaluated). It can be verified that properties (b) and (c) follow from,

$$
\frac{1-e^{-x-1}}{2 x+2}=\frac{e^{x+1}}{2 x+2} \int_{x+1}^{2 x+2} e^{-y} d y \leq g(x) \leq \frac{e^{x+1}}{x+1} \int_{x+1}^{\infty} e^{-y} d y=\frac{1}{x+1} \quad \forall x \geq 0 .
$$

Finally, we also need to show property (d) i.e., $\min _{x \geq 0} f(x)=g(0)$. By definition of $g(\cdot)$ we have, $g(x)-g^{\prime}(x)=\frac{1}{x+1}$. Thus, $\frac{d f(x)}{d x}=0$, which gives us the desired.

Corollary 2. For $g(x)=\beta_{1} \frac{1}{\beta_{2} x+1}$, with $\beta_{1}=0.588, \beta_{2}=0.575$, and $p \leq 0.38$, Algorithm 2 is $(1-O(\sqrt{p})) 0.588$ competitive. 
Proof. Notice that $g(\cdot)$ is strictly decreasing for $x \geq 0$ and $g(0)=\beta_{1}=0.588$. Property (b) and (c) follow by definition. One can verify analytically/numerically that 0 is a minimizer of $f(\cdot)$ (property (d)).

Similarly, it can be verified that the following guarantee holds for the exponential function $g(x)=e^{-x}$ originally proposed in Mehta and Panigrahi (2012).

Corollary 3. For $g(x)=\beta_{1} e^{-\beta_{2} x}$, with $\beta_{1}=0.581, \beta_{2}=0.535$, and $p \leq 0.38$, Algorithm 2 is $(1-O(\sqrt{p})) 0.581$ competitive.

Remarks: In case of vanishing probabilities, the classical upper bound of $(1-1 / e)$ does not necessarily hold when comparing against fully offline or clairvoyant. Unlike the decomposable case, which includes the classical case of unit probabilities, the vanishing probabilities case does not immediately include any previous setting with the $(1-1 / e)$ barrier. More concretely, recall that from the thresholding process perspective, the marginal increase in objective value obtained by matching $i$ to $t$ is approximately $e^{-l_{i}(t)} p_{i t}$. This value decreases over time if $i$ is unsuccessfully matched many times and $l_{i}(t)$ increases. As a consequence of these diminishing marginal gains, the hard instances that lead to a $(1-1 / e)$ upper bound in deterministic settings are not as detrimental here.

\section{Equivalence of PBP and Expectation LP for Large Inventory}

So far, we focused on the case where there is exactly one unit of every offline vertex $i \in I$. An instance where we have (possibly) multiple units $c_{i} \geq 1 \forall i \in I$, is a special case of the single unit setting. Indeed, recall that under the assumption that $c_{i} \rightarrow \infty$ for every $i$, the algorithm of Mehta et al. (2007) is $(1-1 / e)$ competitive against the expectation based LP for arbitrary probabilities. This raises a natural question: Is the path based program PBP equivalent to the expectation based $L P$ for large inventory? In this section, we formally show this to be the case. In fact, we show more strongly that for large inventory, both $O P T(L P)$ and $O P T(P B P)$ are approximately equal to the expected reward of offline (OPT).

Theorem 3. Let $c_{\text {min }}=\min _{i \in I} c_{i}$. For $c_{\text {min }} \geq 2$, we have,

$$
O P T(L P)\left(1-O\left(\sqrt{\frac{\log c_{\min }}{c_{\text {min }}}}\right)\right) \leq O P T \leq O P T(P B P) \leq O P T(L P) .
$$

Hence, for $c_{\min } \rightarrow \infty$, we have, OPT $\rightarrow O P T(L P)$.

Proof. Let $\left\{x_{i t}\right\}_{(i, t) \in E}$ be a feasible solution for the expectation based LP. Consider the nonanticipative offline algorithm that for some carefully chosen value $\delta>0$ (to be decided later), matches every arrival $t \in T$ (in order) by independently sampling a resource according to the 
distribution $\left\{\frac{x_{i t}}{1+\delta}\right\}_{i \in I}$. If the sampled resource is unvailable, the arrival is not matched. We call this the independent rounding (IR) algorithm. Let $I R(\delta)$ denote the expected total reward of this algorithm. Note that $I R(\delta) \leq \mathrm{OPT} \forall \delta \geq 0$. We show that for $\delta=\sqrt{\frac{2 \log c_{\min }}{c_{\min }}}$,

$$
O P T(L P)\left(1-O\left(\sqrt{\frac{\log c_{\min }}{c_{\text {min }}}}\right)\right) \leq I R\left(\sqrt{\frac{2 \log c_{\min }}{c_{\min }}}\right),
$$

which gives us the desired. To prove (24), it suffices to show that in IR, for every edge $(i, t) \in E$, resource $i$ is successfully matched to $t$ w.p. at least $p_{i t} x_{i t}\left(1-O\left(\sqrt{\frac{\log c_{\min }}{c_{\min }}}\right)\right)$. To this end, consider the following indicator random variables for every $(i, t) \in E$, (i) $A(i, t)$ to indicate if $i$ is available at $t$, (ii) $\mathbb{1}(i, t)$ to indicate the success/failure (stochastic reward) of the edge, and (iii) $X_{i t}$ to denote if $i$ is sampled at $t$.

Given an arbitrary edge $(i, t)$, the probability that $i$ is successfully matched to $t$ is

$$
p_{i t} \frac{x_{i t}}{1+\sqrt{\frac{2 \log c_{\min }}{c_{\min }}}} \mathbb{P}\left(\sum_{\tau \leq t} A(i, \tau) \mathbb{1}(i, \tau) X_{i \tau} \leq c_{i}\right),
$$

where,

$$
\begin{aligned}
\mathbb{P}\left(\sum_{\tau \leq t} A(i, \tau) \mathbb{1}(i, \tau) X_{i \tau} \leq c_{i}\right) & \geq \mathbb{P}\left(\sum_{\tau \leq t} \mathbb{1}(i, \tau) X_{i \tau} \leq c_{i}\right) \\
& =1-\mathbb{P}\left(\sum_{\tau \leq t} \mathbb{1}(i, \tau) X_{i \tau}>c_{i}\right) \\
& \geq 1-\frac{1}{\sqrt{c_{\text {min }}}} .
\end{aligned}
$$

The last inequality follows from the standard Chernoff bound for independent Bernoulli random variables. To see this, consider random variables $\left\{Y_{\tau}\right\}_{\tau=1}^{t}:=\left\{\mathbb{1}(i, \tau) X_{i \tau}\right\}_{\tau=1}^{t}$. These are independent Bernoulli random variables (by definition). From the feasibility of $\left\{x_{i t}\right\}_{(i, t) \in E}$, the total mean, $E\left[\sum_{\tau \leq t} Y_{\tau}\right] \leq \frac{c_{i}}{1+\sqrt{\frac{\log c_{\min }}{c_{\min }}}}$. Thus,

$$
\mathbb{P}\left(\sum_{\tau \leq t} Y_{\tau} \geq c_{i}\right) \leq e^{-\frac{\frac{2 \log c_{\min }}{c_{\min }}}{3} c_{i}} \leq \frac{1}{\sqrt{c_{i}}}
$$

\section{Conclusion, Open Problems, and Further Work}

In this paper, we consider a vertex-weighted version of the problem of online matching with stochastic rewards with the goal of designing online algorithms to compete against offline solutions that know the entire sequence of arrivals in advance but learn the stochastic outcomes after matching 
decisions are made. We show that when probabilities $p_{i t}$ decompose as $p_{i} \times p_{t}$ for every edge $(i, t)$, a natural generalization of the Perturbed-Greedy algorithm gives the best possible competitive guarantee of $(1-1 / e)$. When probabilities are small but otherwise fully heterogeneous, we show that a deterministic algorithm that generalizes the Fully-Adaptive algorithm suggested in Mehta and Panigrahi (2012), is 0.596 competitive.

Our analysis for decomposable probabilities involves a novel path based program that gives a tighter bound on the optimal value of offline. For the second case, we develop a new LP free approach where it suffices to find a solution to a certain linear system that compares an online algorithm directly to the actions of offline. We propose a novel candidate solution for this linear system and by generalizing an alternative perspective of the stochastic reward process introduced in Mehta and Panigrahi (2012), we show that the proposed candidate indeed satisfies the linear system. To the best of our knowledge, our results are the first to give a means to obtain provably tighter bound on clairvoyant and fully offline beyond the standard expectation LP. Finally, we show that for arbitrary probabilities but large inventory (many copies of each offline vertex), the path based program and the expectation LP are asymptotically equivalent to clairvoyant.

Beating 1/2 without any assumptions on probabilities still remains open for this problem. Similarly, in the more general setting of online assortments, achieving a competitive guarantee better than 1/2 remains open (except for large inventory, see Golrezaei et al. (2014)). A more immediate line of investigation would be to determine the best one could do in the vanishing probabilities case. Here it may, in fact, be possible to achieve a guarantee better than $(1-1 / e)$. We believe that the ideas of analysis developed here may be useful more broadly in other settings when comparing against offline benchmarks in the presence of stochastic elements. Indeed, Goyal et al. (2021) further generalize the LP free framework, developed in Section 4.3, to circumvent issues with LP based primal-dual analysis for a very different type of post-allocation stochasticity ${ }^{7}$.

\section{Appendix A: Omitted Proofs}

\section{A.1. Missing Proofs from Section 4.1}

Lemma 7. Consider a non-anticipative and deterministic algorithm $\mathcal{A}$. Let $\mathcal{A}(\omega)$ denote the matching output by $\mathcal{A}$ on sample path $\omega \in \Omega$. Similarly, let $\mathcal{A}(\boldsymbol{u})$ denote the matching output by $\mathcal{A}$ on sample path $\boldsymbol{u} \in[0,1]^{n}$ in the threshold viewpoint. For every matching $\mathcal{M}$, we have $\mathbb{P}(\mathcal{A}(\omega)=\mathcal{M})=$ $\mathbb{P}(\mathcal{A}(\boldsymbol{u})=\mathcal{M})$.

\footnotetext{
${ }^{7}$ They consider the case of stochastic reusability, where resources are used for a stochastic duration and then returned for reallocation. The duration is realized only when the resource is returned for reallocation, making it a form of post allocation stochasticity.
} 
Proof. Given a matching $\mathcal{M}$ in the original viewpoint, consider an arbitrary resource $i \in I$ and let $\mathcal{M}_{i}$ denote the ordered set of arrivals matched to $i$. Arbitrarily fix the randomness in all edges not incident on $i$. Let $\omega_{-i}$ denote this (partial) sample path. Conditioned on $\omega_{-i}$, let $p_{\mathcal{M}_{i}}$ denote the probability (w.r.t. randomness in edges incident on $i$ ) that the $\mathcal{M}_{i}$ is the set of arrivals matched to $i$ by $\mathcal{A}$.

Now, conditioned on $\omega_{-i}$, consider the threshold viewpoint just for resource $i$. Generate a random threshold $u_{i}$ for $i$. By definition of the threshold viewpoint, the probability (w.r.t. randomness in $u_{i}$ ) that $\mathcal{A}$ matches $i$ to the ordered set of arrivals $\mathcal{M}_{i}$ in the threshold viewpoint, is exactly $p_{\mathcal{M}_{i}}$. Therefore, we have that sample paths $\left(\omega_{-i}, u_{i}\right)$, where the success/failure of matches made to $i$ are decided by the threshold viewpoint, are stochastically equivalent to sample paths $\omega$. Iteratively applying this argument to covert the sample path of all resources (one at a time), we have that sample paths $\boldsymbol{u}$ and $\omega$ are stochastically equivalent.

Lemma 8. Consider $i \in I, u_{-i} \in[0,1]^{n-1}$, and a deterministic non-anticipative algorithm $\mathcal{A}$. Let $\mathcal{A}_{i}^{u_{-i}}\left(u_{i}\right)$ denote the ordered set of arrivals matched to $i$ in the matching $\mathcal{A}\left(u_{i}, u_{-i}\right)$.

(i) For every $k \in\left[m_{\mathcal{A}}^{u_{-i}}\right]$, $i$ is matched to $t_{k} \in \mathcal{A}_{i}^{u_{-i}}(1)$ if and only if $u_{i}>1-\prod_{j \leq k-1}\left(1-p_{i t_{j}}\right)$. Thus, conditioned on $u_{-i}, i$ is matched to $t_{k}$ with probability $\prod_{j \leq k-1}\left(1-p_{i t_{j}}\right)$.

(ii) For every $u_{i} \in[0,1]$, there exist some $k \in\left[m_{\mathcal{A}}^{u_{-i}}\right]$ such that $\mathcal{A}_{i}^{u_{-i}}\left(u_{i}\right)=\left\{t_{1}, \cdots, t_{k}\right\}$. Further, $\mathcal{A}_{i}^{u_{-i}}\left(u_{i}\right) \subseteq \mathcal{A}_{i}^{u_{-i}}\left(u_{i}^{\prime}\right)$ for every $0 \leq u_{i} \leq u_{i}^{\prime} \leq 1$.

Proof. Given resource $i$ and thresholds $u_{-i}$, let $\mathcal{A}_{i}^{u_{-i}}(1)$ denote the ordered set of arrivals matched to $i$ when $u_{i}=1$. From the non-anticipative nature of $\mathcal{A}$, we have that for every threshold $u_{i} \in[0,1]$, $\mathcal{A}$ sequentially matches $i$ to the arrivals in $\mathcal{A}_{i}^{u_{-i}}(1)$ (in order), until one of the following occurs (i) The probability of success exceeds the threshold $u_{i}$ and $i$ is successfully matched to an arrival in $\mathcal{A}_{i}^{u_{-i}}(1)$, or, (ii) All arrivals in $\mathcal{A}_{i}^{u_{-i}}(1)$ are unsuccessfully matched to $i$ and $i$ is not matched to any other arrivals.

Therefore, for every arrival $t_{k} \in \mathcal{A}_{i}^{u_{-i}}(1), i$ is matched to $t_{k}$ if and only if $u_{i}$ is large enough that the probability of one of $i$ 's previous matches succeeding is smaller than $u_{i}$ i.e., $u_{i}>1-\prod_{j \leq k-1}\left(1-p_{i t_{j}}\right)$. This proves $(i)$. Further, this shows that if $i$ is matched to an arrival $t$ for some threshold value $u_{i}$, then $i$ is matched to $t$ for every threshold value $u_{i}^{\prime} \geq u_{i}$. This proves part $(i i)$.

\section{A.2. Missing Proofs from Section 4.2}

Lemma 9. Given a non-anticipative deterministic algorithm $\mathcal{A}$, resource $i \in I$, threshold vector $u_{-i} \in[0,1]^{n-1}$ and $p \leq 0.38$, we have,

(i) $1-F_{\mathcal{A}}^{u}(x)=\left(1 \pm O\left(\sqrt{p_{i}}\right)\right) e^{-x} \quad \forall x \in\left[0, \frac{1}{\sqrt{p_{i}}}\right]$.

(ii) $F_{\mathcal{A}}^{u_{-i}}(x)=\left(1 \pm O\left(\sqrt{p_{i}}\right)\right)\left(1-e^{-x}\right) \quad \forall x \geq 0$. 
Proof. Consider an arbitrary value $x \geq 0$. Let $\{p(1), \cdots, p(k)\}$ denote the set of all points $\leq x$ in the support of $F_{\mathcal{A}}^{u_{-i}}$. Note that $x-p(k)$ can be at most $p_{i}$. Using the fact that $1-z \leq e^{-z} \leq 1$ for $z \geq 0$, we have that, $e^{-p(k)}=\left(1 \pm O\left(p_{i}\right)\right) e^{-x}$. Let $p(0)=0$ and $p_{i t_{j}}=p(j)-p(j-1) \quad \forall j \in[k]$.

Proof of $(i)$ : By definition of distribution $F_{\mathcal{A}}^{u_{-i}}$, we have, $1-F_{\mathcal{A}}^{u_{-i}}(x)=\prod_{j=1}^{k}\left(1-p_{i t_{j}}\right)$. We show that for $x \in\left[0, \frac{1}{\sqrt{p_{i}}}\right]$, the product $\prod_{j=1}^{k}\left(1-p_{i t_{j}}\right)$ is well approximated by $e^{-p(k)}$. Then, the fact that $e^{-p(k)}=\left(1 \pm O\left(p_{i}\right)\right) e^{-x}$, gives us the desired.

$$
\begin{aligned}
1 \leq \frac{e^{-\sum_{j=1}^{k} p_{i t_{j}}}}{\prod_{j=1}^{k}\left(1-p_{i t_{j}}\right)} & \stackrel{(a)}{\leq} \frac{\prod_{j=1}^{k}\left(1-p_{i t_{j}}+p_{i t_{j}}^{2}\right)}{\prod_{j=1}^{k}\left(1-p_{i t_{j}}\right)} \\
& =\frac{\prod_{j=1}^{k}\left(1-p_{i t_{j}}\right) \prod_{j=1}^{k}\left(1+\frac{p_{i t_{j}}^{2}}{1-p_{i}}\right)}{\prod_{j=1}^{k}\left(1-p_{i t_{j}}\right)} \\
& \stackrel{(b)}{\leq} \prod_{j=1}^{k} e^{\frac{p_{i}}{1-p_{i}} p_{i t_{j}}} \leq e^{\frac{p_{i}}{1-p_{i}}\left(\frac{1}{\sqrt{p_{i}}}\right)} \\
& \stackrel{(c)}{\leq} 1+O\left(\sqrt{p_{i}}\right)
\end{aligned}
$$

here $(a)$ follows from the inequality, $e^{-z} \leq 1-z+z^{2}$ for $z \geq 0$. Inequality (b) follows from the fact that $1+z \leq e^{z}$ for $z \in \mathbb{R}$. To see $(c)$, observe that for $p \leq 0.38$, we have, $\frac{\sqrt{p_{i}}}{1-p_{i}} \leq 1$. Then, using the inequality $e^{z} \leq 1+2 z$ for $z \in[0,1]$, gives us the final inequality.

Proof of $($ ii $)$ : From part $(i)$ we have,

$$
F_{\mathcal{A}}^{u_{-i}}(x)=\sum_{j=1}^{k}\left[p_{i t_{j}} \prod_{\ell=1}^{j}\left(1-p_{i t_{\ell}}\right)\right]=\left(1 \pm O\left(\sqrt{p_{i}}\right)\right) \sum_{j=1}^{k} p_{i t_{j}} e^{-p(j)} \quad \forall x \in\left[0, \frac{1}{\sqrt{p_{i}}}\right] .
$$

Notice that $\sum_{j=1}^{k} p_{i t_{j}} e^{-p(j)}=\left(1 \pm O\left(p_{i}\right)\right) \int_{0}^{x} e^{-z} d z$, which establishes part $(i i)$ for $x \leq \frac{1}{\sqrt{p_{i}}}$. Consequently, we have, $F_{\mathcal{A}}^{u_{-i}}\left(\frac{1}{\sqrt{p_{i}}}\right) \geq\left(1-O\left(\sqrt{p_{i}}\right)\right)$.

For $x>\frac{1}{\sqrt{p_{i}}}$, we have $F_{\mathcal{A}}^{u_{-i}}\left(\frac{1}{\sqrt{p_{i}}}\right) \leq F_{\mathcal{A}}^{u_{-i}}(x) \leq 1$. Then, using $F_{\mathcal{A}}^{u_{-i}}\left(\frac{1}{\sqrt{p_{i}}}\right) \geq\left(1-O\left(\sqrt{p_{i}}\right)\right)$, we get, $F_{\mathcal{A}}^{u_{-i}}(x)=\left(1 \pm O\left(\sqrt{p_{i}}\right)\right)\left(1-e^{-x}\right) \quad \forall x \geq 0$.

Lemma 10. Consider a deterministic non-anticipative algorithm $\mathcal{A}$, resouce $i \in I$, threshold vector $u_{-i} \in[0,1]^{n-1}$, a non-decreasing integrable function $h: \mathbb{R}^{+} \rightarrow \mathbb{R}^{+}$such that,

(i) $h(0)=0$,

(ii) $h(x)=h\left(\frac{1}{\sqrt{p_{i}}}\right) \forall x \geq \frac{1}{\sqrt{p_{i}}}$.

For $p \leq 0.38$, we have,

$$
E_{u_{i}}\left[h\left(G_{\mathcal{A}}^{u_{-i}}\left(u_{i}\right)\right)\right]=\left(1 \pm O\left(\sqrt{p_{i}}\right)\right) E_{b \sim \operatorname{Exp}(1)}[h(b)] .
$$


Proof. Let $p(0)=0$ and recall that $p(\ell)=p\left(m_{\mathcal{A}}^{u_{-i}}\right)+\left(\ell-m_{\mathcal{A}}^{u_{-i}}\right) p_{i} \quad \forall \ell \geq m_{\mathcal{A}}^{u_{-i}}$. For simplicity, let $\Delta h(k)=h(p(k))-h(p(k-1)) \quad \forall k \geq 1$. Using the fact that $h(x)=h\left(\frac{1}{\sqrt{p_{i}}}\right)$ for all $x \geq 1 / \sqrt{p_{i}}$, we have, $\Delta h(k)=0$ for every $k$ such that $p(k-1) \geq \frac{1}{\sqrt{p_{i}}}$.

$$
\begin{aligned}
E_{u_{i}}\left[h\left(G_{\mathcal{A}}^{u_{-i}}\left(u_{i}\right)\right)\right] & =E_{b \sim F_{\mathcal{A}}^{u}}[h(b)], \\
& =\sum_{k \geq 1}\left(1-F_{\mathcal{A}}^{u_{-i}}(p(k-1))\right) \Delta h(k), \\
& =\left(1 \pm O\left(\sqrt{p_{i}}\right)\right) \sum_{k \geq 1} e^{-p(k-1)} \Delta h(k), \\
& =\left(1 \pm O\left(\sqrt{p_{i}}\right)\right) \sum_{k \geq 1}\left(e^{-p(k-1)}-e^{-p(k)}\right) h(p(k)), \\
& =\left(1 \pm O\left(\sqrt{p_{i}}\right)\right) \sum_{k \geq 1}\left(h(p(k)) \int_{p(k-1)}^{p(k)} e^{-x} d x\right),
\end{aligned}
$$

here the first and second equation follow from the definition of $F_{\mathcal{A}}^{u_{-i}}$. The third equation follows from Lemma $9(i)$ and the fact that $\Delta h(k)=0$ for every $k$ such that $p(k-1) \geq \frac{1}{\sqrt{p_{i}}}$.

Now, to prove the main claim it suffices to show that,

$$
\sum_{k \geq 1}\left(h(p(k)) \int_{p(k-1)}^{p(k)} e^{-x} d x\right)=\left(1 \pm O\left(p_{i}\right)\right) \int_{0}^{+\infty} e^{-x} h(x) d x .
$$

Observe that for every non-decreasing and integrable function $h(\cdot)$,

$$
\int_{p(k-1)}^{p(k)} e^{-x} h(x) d x \leq h(p(k)) \int_{p(k-1)}^{p(k)} e^{-x} d x \leq e^{p_{i}} \int_{p(k-1)+p_{i}}^{p(k)+p_{i}} e^{-x} h(x) d x \quad \forall k \geq 1 .
$$

Summing up the inequalities above for $k \geq 1$ and using the fact that $e^{p_{i}} \leq 1+2 p_{i}$ for $p_{i} \in[0,1]$, completes the proof.

Lemma 1. The optimal fully offline and clairvoyant algorithms are deterministic and given by a (possibly exponential time) dynamic program.

Proof. Let us start with clairvoyant benchmark. The state space is given by the set of arrivals matched and the success/failure of matches so far. The decision space of clairvoyant is simply the matching decision for the next arrival in the sequence. Let $V\left(t, S_{t}\right)$ denote the optimal value-to-go given that at arrival $t$, the set of remaining resources is $S_{t}$. Clearly,

$$
V\left(t, S_{t}\right)=\max _{i \mid i \in S_{t},(i, t) \in E} p_{i t}\left(r_{i}+V\left(t+1, S_{t} \backslash\{i\}\right)\right)+\left(1-p_{i t}\right) V\left(t+1, S_{t}\right) .
$$

Therefore, regardless of the values of $V\left(t+1, S_{t}\right)$ and $V\left(t+1, S_{t} \backslash\{i\}\right)$, the optimal decision at arrival $t$ is deterministic. In case of fully offline, the action space also includes the order in which arrivals are visited. It is easy to see that a similar argument applies to fully offline. 


\section{A.3. Missing Proofs from Section 4.4}

LEMMA 12. For every $i \in I$, let $\mathrm{OPT}_{i}$ and $\mathrm{OPT}_{i}^{\text {tr }}$ denote the expected total revenue from matching resource $i$ in $\mathrm{OPT}$ and in $\mathrm{OPT}^{t r}$ respectively. Then, we have, $\mathrm{OPT}_{i}^{t r} \geq(1-O(\sqrt{p})) \mathrm{OPT}_{i} \forall i \in I$.

Proof. Consider an arbitrary resource $i \in I$ and fix sample path $u_{-i} \in[0,1]^{n-1}$. Let $p\left(m_{\mathrm{OPT}}^{u_{-i}}\right)$ and $p\left(m_{\mathrm{OPT}^{t r}}^{u_{-}}\right)$denote the effort threshold of $i$ in OPT and OPT ${ }^{t r}$ respectively. By definition of $\mathrm{OPT}^{t r}$, we have, $p\left(m_{\mathrm{OPT}^{t r}}^{u_{-i}}\right)=\min \left\{p\left(m_{\mathrm{OPT}}^{u_{-i}}\right), \frac{1}{\sqrt{p}}\right\}$. Let $p_{1}$ denote the probability that $i$ is successfully matched in OPT (conditioned on $u_{-i}$ ). Similarly, let $p_{2}$ denote the probability that $i$ is successfully matched in $\mathrm{OPT}^{t r}$. It suffices to show that $p_{2} \geq(1-O(\sqrt{p})) p_{1}$.

When $p\left(m_{\mathrm{OPT} t r}^{u_{-i}}\right)=p\left(m_{\mathrm{OPT}}^{u_{-i}}\right)$, we have, $p_{2}=p_{1}$, since OPT and OPT ${ }^{t r}$ match $i$ to the same set of arrivals. If $p\left(m_{\mathrm{OPT}^{t r}}^{u_{-i}}\right)<p\left(m_{\mathrm{OPT}}^{u_{-i}}\right)$, then we have, $p\left(m_{\mathrm{OPT}^{t r}}^{u_{-i}}\right)=\frac{1}{\sqrt{p}}$. Using Corollary $1(i i)$ and Lemma $9(i i)$, we have that, $p_{2} \geq\left(1-O\left(\sqrt{p_{i}}\right)\right) e^{-\frac{1}{\sqrt{p}}} \geq 1-O(\sqrt{p})$. Since $p_{1} \leq 1$, we have the desired.

\section{Appendix B: Comparison with Configuration LP in Huang and Zhang (2020)}

Recall that Huang and Zhang (2020) show a 0.576 competitive ratio guarantee against the expectation LP for vanishingly small probabilities. The use a primal-dual approach with a new LP that approximates the expectation LP for small probabilities. The dual of the LP in Section 2.2 of Huang and Zhang (2020), has the following constraints (paraphrased in our notation),

$$
\theta_{i}+\sum_{t \in N_{i}} \lambda_{t} \geq \alpha r_{i} \min \left\{1, \sum_{t \in N_{i}} p_{i t}\right\} \quad \forall i, N_{i} \subseteq\{t \mid(i, t) \in E\}
$$

These constraints differ from inequalities (13) in our LP free framework in two important ways: (i) For any given $i$, the above constraints are imposed for all subsets of edges incident on $i$. In contrast, inequalities (13) are imposed over all possible sample paths $u_{-i}$. The dependence on sample paths allows us to define our candidate solution as a function of coupled randomness in ALG and OPT.

(ii) The quantity $\min \left\{1, \sum_{t \in N_{i}} p_{i t}\right\}$ is, in general, a loose upper bound on $\mathrm{OPT}_{i}$, even for small edge probabilities. Recall that in $(13)$, we approximate $\mathrm{OPT}_{i}$ with $r_{i} E_{u_{-i}}\left[\left(1-e^{p\left(m_{\mathrm{OPT}}^{u_{-i}}\right)}\right)\right]$.

\section{Acknowledgments}

We would like to thank anonymous referees for their valuable feedback. We also thank Will Ma for a helpful discussion on the results in Mehta and Panigrahi (2012). This research was partially supported by NSF Grant CMMI 1636046 and NSF CAREER Award CMMI 1351838.

\section{References}

Acimovic J, Farias VF (2019) The fulfillment-optimization problem. Operations Research 8 Management Science in the Age of Analytics, 218-237 (INFORMS). 
Aggarwal G, Goel G, Karande C, Mehta A (2011) Online vertex-weighted bipartite matching and singlebid budgeted allocations. Proceedings of the twenty-second annual ACM-SIAM symposium on Discrete Algorithms, 1253-1264 (SIAM).

Aouad A, Saritaç Ö (2020) Dynamic stochastic matching under limited time. Biró P, Hartline JD, Ostrovsky M, Procaccia AD, eds., EC '20: The 21st ACM Conference on Economics and Computation, Virtual Event, Hungary, July 13-17, 2020, 789-790 (ACM).

Ashlagi I, Burq M, Dutta C, Jaillet P, Saberi A, Sholley C (2019) Edge weighted online windowed matching. Karlin A, Immorlica N, Johari R, eds., Proceedings of the 2019 ACM Conference on Economics and Computation, EC 2019, Phoenix, AZ, USA, June 24-28, 2019, 729-742 (ACM).

Bansal N, Gupta A, Li J, Mestre J, Nagarajan V, Rudra A (2010) When lp is the cure for your matching woes: Improved bounds for stochastic matchings. European Symposium on Algorithms, 218-229 (Springer).

Birnbaum B, Mathieu C (2008) On-line bipartite matching made simple. ACM SIGACT News 39(1):80-87.

Borodin A, MacRury C, Rakheja A (2020) Bipartite stochastic matching: Online, random order, and i.i.d. models. ArXiv abs/2004.14304.

Buchbinder N, Jain K, Naor JS (2007) Online primal-dual algorithms for maximizing ad-auctions revenue. European Symposium on Algorithms, 253-264 (Springer).

Chan CW, Farias VF (2009) Stochastic depletion problems: Effective myopic policies for a class of dynamic optimization problems. Mathematics of Operations Research 34(2):333-350.

Charikar M, Henzinger M, Nguyen HL (2014) Online bipartite matching with decomposable weights. European Symposium on Algorithms, 260-271 (Springer).

Devanur NR, Hayes TP (2009) The adwords problem: online keyword matching with budgeted bidders under random permutations. Proceedings of the 10th ACM conference on Electronic commerce, 71-78 (ACM).

Devanur NR, Jain K, Kleinberg RD (2013) Randomized primal-dual analysis of ranking for online bipartite matching. Proceedings of the twenty-fourth annual ACM-SIAM symposium on Discrete algorithms, 101-107 (Society for Industrial and Applied Mathematics).

Feng Y, Niazadeh R, Saberi A (2019) Linear programming based online policies for real-time assortment of reusable resources. Available at SSRN 3421227.

Feng Y, Niazadeh R, Saberi A (2021) Two-stage stochastic matching with application to ride hailing. Marx D, ed., Proceedings of the 2021 ACM-SIAM Symposium on Discrete Algorithms, SODA 2021, Virtual Conference, January 10 - 13, 2021, 2862-2877 (SIAM).

Goel G, Mehta A (2008) Online budgeted matching in random input models with applications to adwords. Proceedings of the nineteenth annual ACM-SIAM symposium on Discrete algorithms, 982-991 (Society for Industrial and Applied Mathematics).

Golrezaei N, Nazerzadeh H, Rusmevichientong P (2014) Real-time optimization of personalized assortments. Management Science 60(6):1532-1551. 
Gong XY, Goyal V, Iyengar G, Simchi-Levi D, Udwani R, Wang S (2019) Online assortment optimization with reusable resources. Available at SSRN 3334789 .

Goyal V, Iyengar G, Udwani R (2021) Asymptotically optimal competitive ratio for online allocation of reusable resources. arXiv preprint arXiv:2002.02430 .

Grammel N, Brubach B, Ma W, Srinivasan A (2021) Follow your star: New frameworks for online stochastic matching with known and unknown patience 130:2872-2880.

Ho CJ, Vaughan JW (2012) Online task assignment in crowdsourcing markets. AAAI, volume 12, 45-51.

Huang Z, Zhang Q (2020) Online primal dual meets online matching with stochastic rewards: Configuration lp to the rescue. arXiv preprint arXiv:2002.01802 .

Kalyanasundaram B, Pruhs KR (2000) An optimal deterministic algorithm for online b-matching. Theoretical Computer Science 233(1-2):319-325.

Karger DR, Oh S, Shah D (2014) Budget-optimal task allocation for reliable crowdsourcing systems. Operations Research 62(1):1-24.

Karp RM, Vazirani UV, Vazirani VV (1990) An optimal algorithm for on-line bipartite matching. Proceedings of the twenty-second annual ACM symposium on Theory of computing, 352-358 (ACM).

Le Cam L (1960) An approximation theorem for the poisson binomial distribution. Pacific Journal of Mathematics 10(4):1181-1197.

Ma W, Simchi-Levi D (2018) Online resource allocation under arbitrary arrivals: Optimal algorithms and tight competitive ratios. https://ssrn.com/abstract=2989332 .

Mehta A, Panigrahi D (2012) Online matching with stochastic rewards. Foundations of Computer Science (FOCS), 2012 IEEE 53rd Annual Symposium on, 728-737 (IEEE).

Mehta A, Saberi A, Vazirani U, Vazirani V (2007) Adwords and generalized online matching. J. ACM 54(5):22-es, ISSN 0004-5411.

Mehta A, Waggoner B, Zadimoghaddam M (2015) Online stochastic matching with unequal probabilities. Proceedings of the twenty-sixth annual ACM-SIAM symposium on Discrete algorithms, 1388-1404 (Society for Industrial and Applied Mathematics).

Mehta A, et al. (2013) Online matching and ad allocation. Foundations and Trends@ in Theoretical Computer Science 8(4):265-368.

Mirrokni VS, Gharan SO, Zadimoghaddam M (2012) Simultaneous approximations for adversarial and stochastic online budgeted allocation. Proceedings of the twenty-third annual ACM-SIAM symposium on Discrete Algorithms, 1690-1701 (Society for Industrial and Applied Mathematics).

Udwani R (2021) Adwords with unknown budgets. arXiv preprint arXiv:2110.00504 . 